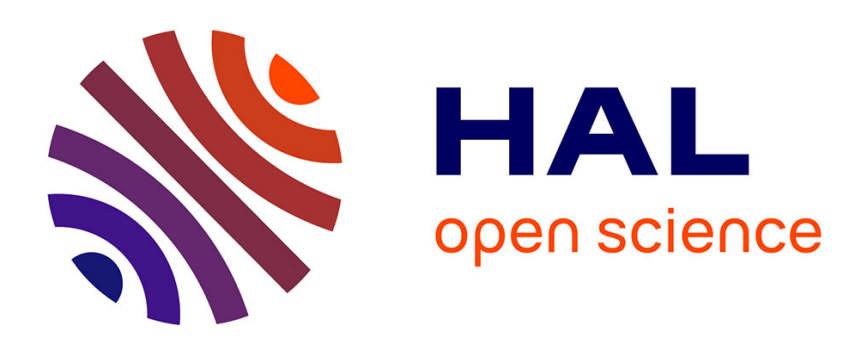

\title{
A Variable-Length Cell Transmission Model for Road Traffic Systems
}

Carlos Canudas de Wit, Antonella Ferrara

\section{To cite this version:}

Carlos Canudas de Wit, Antonella Ferrara. A Variable-Length Cell Transmission Model for Road Traffic Systems. Transportation research. Part C, Emerging technologies, 2018, 97, pp.428-455. 10.1016/j.trc.2018.07.023 . hal-02268191

\section{HAL Id: hal-02268191 \\ https://hal.science/hal-02268191}

Submitted on 20 Aug 2019

HAL is a multi-disciplinary open access archive for the deposit and dissemination of scientific research documents, whether they are published or not. The documents may come from teaching and research institutions in France or abroad, or from public or private research centers.
L'archive ouverte pluridisciplinaire HAL, est destinée au dépôt et à la diffusion de documents scientifiques de niveau recherche, publiés ou non, émanant des établissements d'enseignement et de recherche français ou étrangers, des laboratoires publics ou privés. 


\title{
A Variable-Length Cell Transmission Model for Road Traffic Systems
}

\author{
Carlos Canudas-de-Wit*, and Antonella Ferrara
}

\begin{abstract}
In this paper we propose a new aggregated Variable-Length cell transmission Model (VLM) for traffic systems consisting of only three lumped state variables. The first two state variables are the lumped (or averaged) densities associated with the two cells of variable length into which the considered road section is subdivided. These cells are, respectively, a downstream congested cell, of length $l$, and an upstream free cell, of length $L-l, L$ being the total length of the section. The third state, namely $l$, describes the position of the congestion wave front. The paper, apart from providing the new traffic model formulation, also investigates several mathematical properties of this model, ensuring the consistency with respect to its inherent mass (or vehicle) conservation law. Two case studies, a ring road and a urban road with traffic lights, are considered to assess the proposed model validity also relying on simulation evidence.
\end{abstract}

Keywords. Road Traffic models, Cell-Transmission model, Variable-length model.

\section{INTRODUCTION}

Physicists, mathematicians and engineers have developed models of vehicular traffic for almost fifty years [25], [26], [27], [44]. Models differ and can be classified on the basis of the level of detail they adopt in describing traffic phenomena. An enlightening and well documented overview of the development of traffic flow models in a form of a model tree is given in [45]. They classify traffic models in the four well identified families: the fundamental relation, microscopic, mesoscopic and macroscopic models.

Selecting a particular model depends on the type of usage. For traffic operation and planning, a coarse representation of traffic suffices, for instance by resorting to models only describing

* Carlos Canudas-de-Wit (contact author). CNRS, Department of Automatic Control, GIPSA-lab, Grenoble, France. carlos.canudas-de-wit@gipsa-lab.fr

$\diamond$ Antonella Ferrara, Dipartimento di Ingegneria Industriale e dell'Informazione, University of Pavia, Italy 
the stationary traffic behaviour [6], [37]. In contrast, for forecasting traveling times and for traffic control purposes, mesoscopic or macroscopic dynamical models are often preferred as they capture the traffic evolution in time and space necessary for dynamic traffic assignment and for control design. However, There are fundamental differences in the type of model choice when the models are used either for traffic prediction or for traffic control.

For real-time traffic prediction, models with high-refinement level are often preferred as they can be run in "open-loop" and can be efficiently coded either as "algorithms" or as numerical time/space discretization of PDEs or/and ODEs representing the traffic evolution. They can be mesoscopic or macroscopic, and eventually include multi-classes and complex specific components for optimal merging/diverging phases modeling. Finally they can include "high-frequency" components (i.e.stop-and-go behaviour) for instance by adding new variables (i.e. velocities) to the model in addition to the standard densities or traveling time. Among highorder models, the generic second order models family originally proposed in [31] is a good example covering many other special cases of second-order models.

In contrast to the traffic prediction, model for control design require different attributes which are less addressed in the literature on traffic modelling, but are of crucial importance for modelbased traffic control design (see, e.g., [8]). They should:

i) be consistent with the actuator dynamics,

ii) have reduced/limited complexity, and

iii) be described in closed-forms

Let illustrate such a characteristics.

i) Actuator dynamic consistency implies that the model does not need to capture higher frequencies beyond the frequency response of the selected actuator. For instance, in the case of variable speed limit control (VSC), the change of rate (in time and space) of the "controlled" speed limit cannot be modified too fast (order of several minutes), otherwise the drives cannot follow such regulated speed limitations. Therefore high-frequency effects (i.e. stop-and-go highfrequency waves which occur at frequencies of less than a minute) produced during congestion cannot be attenuated by this mean (but it may be if the car speeds are controlled individually). However, the "low-frequency" (or average) component of the shockwave can be indeed effectively influenced by VSC or other similar control action like ramp metering. Under such actuator limitations, first-order macroscopic models are preferable as models to be used for control design purposes. A popular example of such a macroscopic model is the LWR model by Lighthill and 
Whitham [34], and Richards [41] which describes the evolution of traffic over time and space relying on the analogy between traffic evolution and flows dynamics in fluids. Similarly, when it comes to controlling traffic lights in urban networks, only the "average" or "aggregate" evolution of the road densities counts, as the traffic light operates on a time-basis of about $100 \mathrm{~s}$ which is not enough to cope with high-frequency congestion effects.

ii) Reduced/limited complexity refers to the capacity of a model to capture the main effect to be controlled while preserving a certain degree of simplicity in its use. For instance, the LWR which satisfies property $i$ ), so as its discretized in space counterpart named the the Cell Transmission Model (CTM) [14], [30], well captures the "low-frequency" traffic evolution. In particular, resolving the CTM (in open-loop) is straightforward and not computationally difficult, even if many small cells are considered. However, using the the CTM for model predictive closed-loop control [12], [16], [17], [22] may results in a NP-complete optimization problem in particular when large optimization time-horizon and many cell are considered. Specifically, when using the CTM for control, the model is represented in its state-space switching form:

$$
x(k+1)=A(x(k)) x(k)+B(x(k)) u(k)
$$

Where $x(k) \geq 0$ is the $n$-dimensional vector of all densities in the $n$-cells, $u(k)$ are the boundary (demands/supply) flows and $A(x(k)), B(x(k))$ are state-dependent matrices with coefficient dependent on physical parameters of the system (FD, split ratios, etc.). The number of possible instances of those matrix is $2^{n-1}$. For large-scale traffic networks, the system state increases substantially and its combinatory nature may render the on-line model-based optimal control design highly-complex, and, as such, incompatible with the online implementation, even for short time-horizon. These considerations strongly motivates the search for models with further level of state aggregation.

iii) Models with closed forms are "explicit" mathematic expressions, such as differential or finite difference equations, that can be solved using only information about initial conditions (i.e. initial densities) and external inputs (boundary demands and supplies). Note that there are some other models that are "implicit" in the sense that one part of the model is described by some optimization process and not by explicit differential or partial equations.

Closed forms are suited for control design as they allows for analysis of the specific control properties of the model like: equilibrium points, stability of the equilibria, controllability of 
the state variables, etc. They are also necessary for the control synthesis since they enables to determine the explicit expression of the associated feedback laws.

The model presented in this paper belongs to the class of models for control design with the attributes mentioned before. It is not intended as a general purpose model (it does not model separate lines, neither multi-modalities for instance), but it can be used in scenarios where control design is fundamental, such as when bottlenecks are present in highways, as well as in urban networks inclined to congest. It is derived with the aim of complying with considerations $i$ ) $-i i i)$.

Having in mind the large variety of traffic models published in the literature, it is useful to briefly review some of the models that were proposed in the literature with the same spirit of the model presented in this paper, making reference in particular to the aggregation aspect addressed in ii). They are first-order models having limited complexity, and link models with an explicit modelling of queues.

\section{A. Examples of first-order models with limited complexity and explicit queue modeling}

One natural way to limit the model complexity is to reduce the variables number by using "averaged" variables that aggregate the behaviour of some particular areas of the road. For instance by modelling the "queues" (areas that are congested) and their propagation along the network instead of modelling the whole density distribution as in the LWR, or modelling many small cells as in the CTM.

A possible way to do this is to describe the LWR dynamics at the level of a "link" in terms of cumulative vehicle numbers at the link end, rather than using a fine spatial discretization as proposed by the CTM. Such a model was proposed in the original works of Yperman and coworkers [48] and was named Link Transmission Model (LTM), making reference to the transposition from cell in the CTM to the one-cell version in the LTM. The authors showed that this process reduce the model complexity of a factor proportional to the number of cell in the CTM. Several extensions to the basic version of LTM have been proposed, including the use of a sort of vertical virtual queue to model intersection delays. The LTM can also be conveniently adopted to model multi-commodity traffic systems [46], [47]. Its more recent versions have overcome possible limitations to temporal discretization due to computational reasons of the basic formulation [24].

Store and forward models (SFMs) are simple models integrating the difference between the total inflows and outflows in a link. The first SFM traces back to the original works of Gazis 
and Potts (1963) [18] and has been used in various subsequent works, especially for road traffic control optimization [1]. The model states describe the cumulated number of vehicles in a link. The SFM introduces several simplifications (i.e. model linearization, average of the traffic light duty cycle, manual track of the queue bounds, etc. ) at the cost of the loss of many of the model original kinematic properties (shock wave propagation, boundedness of the solutions, etc.).

Similar queue models inspired by data transmission have also been proposed in the literature and used for control design. Those models aims to describe the "queue" evolution in a link by integrating the differences between inflows and outflows. However, as in the case of the SFM, the queue models are "autonomous" as they depends only on the link queue state and not on the state of the neighbor links. In some studies, the vehicle inflows and outflows are described by time-dependent functions that, in some cases, are assumed to be stochastic [49].

A common limitation in many of the SFMs is that the queues are "vertical" with unlimited capacity, which does not permit saturation of a downstream link that blocks the movement of vehicles from an upstream link. This may result in motion-blocking problems [49], [2], and in a substantial deviation from waves propagations as predicted by the original LWR model.

A possible alternative is the Two-regime transmission model (TTM) based on the first-order traffic flow theory. The TTM is designed to be efficient and accurate enough for planning purposes, as the authors clearly claim [3]. The TTM considers the time-dependent density states of network links over two regimes namely, free-flowing and congested regimes, and dynamically models the time-dependent queue length, but without splitting the link into cells. The model is not expressible in closed form but tracks the time-space evolution of the in/out flows relying on the solutions of the wave propagation equation.

Note that with this brief overview we do not pretend to have surveyed the entire bunch of literature on vehicular traffic modelling. As previously mentioned, the aim here was only to mention some models with similar features with respect to those of the model presented in this paper. A more comprehensive comparison among different models, relying on simulated and possibly real data, will be done in a future work.

\section{B. Paper contribution}

The model proposed in the present paper is an aggregated Variable-Length cell transmission Model (VLM) based in some of the ideas originally introduced in [9]. Its derivation results from 
averaging the density in the original LWR model and ensuring that the wave propagations are suitable considered.

In the proposed VLM each link is described by three main lumped state variables. The first two state variables are the lumped (or averaged) densities associated with the two cells of variable length into which the considered road section is subdivided. These cells are, respectively, a downstream congested cell, of length $l$, and an upstream free cell, of length $L-l, L$ being the total length of the section. The third state, namely $l$, describes the position of the congestion wave front. The model also consider specific singular cases when the link is either fully congested or fully free. As the model is given in closed-form, the mathematical properties of the model can be formally addressed. Among these, the consistency with respect to its inherent mass (or vehicle) conservation law, and the capability of accurately describing the occurrence and propagation of both shock and rarefaction waves not only inside of the link (this is an intrinsic property), but also between links. The paper also analyses several stability and invariant properties of the model fundamental for control design. In that the model satisfies all the desired attributes for the control design. Finally, the structure of the model is indeed adaptable to several modeling and control scenarios that are briefly described at the end of the paper.

The paper is organized as follows. Some preliminary issues on the continuous LWR model and the continuous-time formulation of the CTM are reported in Section II. The new VariableLength Cell model is presented in Section III and its mathematical properties are discussed in Section IV. The proposed model is further developed to be able to correctly describe rarefaction waves, thus attaining the whole model formulation reported in Section V. A discussion of the proposed model features versus those of the classical LWR traffic flow model and the standard Cell Transmission Model has been provided in Section VI, also focusing on the capability of the different models to describe the evolution of shock-waves. The proposed model is then assessed through two case studies analyzed in Section VII: a ring road and a urban road with traffic lights, discussed in Subsection VII-A and Subsection VII-B, respectively, also relying on simulation evidence. The two case studies provide a demonstration of how the model can be conveniently used for optimal control design. Some conclusions are finally reported in Section VIII. 


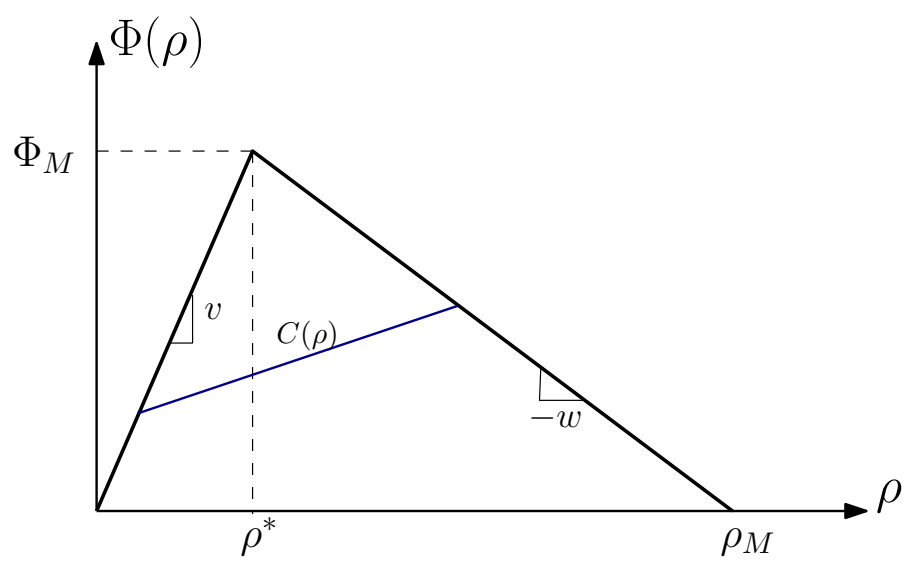

Fig. 1. Schematic representation of the triangular piece-wise fundamental diagram, where $\varphi_{M}$ is the the maximum flow, $\rho_{M}$ is the maximum density, $\rho^{*}$ is the critical density, $v$ is the free-flow velocity, and $-w$ is the congested velocity. $C(\rho)$ is the chord used to study the Oleinik's entropy condition (50)

\section{PRELIMINARIES}

The simplest continuous macroscopic traffic model is the so-called LWR model, proposed by Lighthill, Whitham [34], and Richards [41]. It is based on the vehicle conservation's principle, and on the assumption that the traffic can be described by the empiric relation between the flow, $\varphi$, and density, $\rho$, as: $\varphi=\Phi(\rho)$, where the function $\Phi(\cdot)$ is called Fundamental Diagram. The constitutive assumption of this model, motivated by experimental data, is that the vehicles tend to travel at an equilibrium speed and that $\varphi=V(\rho) \rho$, where $V(\rho)$ is the flow speed depending on the density. $V(\rho)$ varies in the range $[0, v]$, where $v$ is the maximum velocity at free-flow. As shown in Fig. 1, the fundamental diagram can be defined, in its simplest form, as a triangle with its maximum at $\varphi_{M}=\Phi\left(\rho^{*}\right)$ describing the maximum capacity of the road. The critical density $\rho^{*}$ defines the boundary between the decongested and the congested modes, while $\rho_{M}$ is the maximum density that the road can withstand. The slope $-w$ defines the speed at which congestion travels upstream.

The evolution of the number of vehicles, $N$, within any spatial section $(0, L)$ is given by the following mass (vehicles) conservation law

$$
\frac{\mathrm{d}}{\mathrm{d} t} N=\varphi_{\text {in }}-\varphi_{\text {out }}, \quad N=\int_{0}^{L} \rho(x, t) \mathrm{d} x
$$


where $\varphi_{\text {in }}$ and $\varphi_{\text {out }}$ are the input (at $x=L$ ) and output (at $x=0$ ) flows at the boundaries of the road section of length $L$. Equation (1) can be rewritten (see [34]) as a hyperbolic equation involving only the density

$$
\partial_{t} \rho+\partial_{x} \Phi(\rho)=\partial_{t} \rho+\partial_{\rho} \Phi \cdot \partial_{x} \rho=0
$$

The macroscopic continuous density dynamics is then given by the LWR Cauchy problem described by (2) with the initial condition $\rho(x, 0)=\rho^{0}(x)$. The model has been shown to be consistent with hydrodynamic theory [14]. Validation tests with real data have been reported in $[35]$.

\section{A. Cell-Transmission Model: continuous-time formulation}

Assume now that the road section is subdivided into $n$-cells of constant length $\Delta l_{i}$. Let us denote by $\rho_{i}$ the density of the $i^{t h}$ cell of the section. Then, the number of vehicles per cell can be computed as $N_{i}=\rho_{i} \Delta l_{i}$.

As conservation laws generate irregular flows, they cannot be integrated numerically using standard methods (see [14], [32]). An efficient first-order numerical method to treat such conservation laws is the Godunov scheme [19]. It reproduces correctly the propagation of the shock waves, avoiding oscillating behavior and having a physical interpretation. Using the Godunov mathematical formalism, the conservation law (1) takes the following form, which can be regarded as the continuous-time version of the discrete-time Cell-Transmission Model (CTM), see [14], [30]

$$
\begin{aligned}
\dot{\rho}_{1}(t) & =\frac{1}{\Delta l_{1}}\left(\varphi_{\text {in }}(t)-\varphi_{1}(t)\right) \\
\dot{\rho}_{2}(t) & =\frac{1}{\Delta l_{2}}\left(\varphi_{1}(t)-\varphi_{2}(t)\right) \\
& \vdots \\
\dot{\rho}_{i}(t) & =\frac{1}{\Delta l_{i}}\left(\varphi_{i-1}(t)-\varphi_{i}(t)\right) \\
& \vdots \\
\dot{\rho}_{n}(t) & =\frac{1}{\Delta l_{n}}\left(\varphi_{n-1}(t)-\varphi_{\text {out }}(t)\right)
\end{aligned}
$$

where $\varphi_{i}$ is the interface flow between the cells $i$ and $i+1$ given as

$$
\varphi_{i}=\min \left\{D_{i}, S_{i+1}\right\}
$$


with

$$
\begin{aligned}
D_{i} & =\min \left\{v_{i} \rho_{i}, \varphi_{M, i}\right\}, \\
S_{i+1} & =\min \left\{\varphi_{M, i+1}, w_{i+1}\left(\rho_{M, i+1}-\rho_{i+1}\right)\right\}
\end{aligned}
$$

where the demand $D_{i}$ is the flow that can be delivered by the cell $i$ to the cell $i+1$, while the supply $S_{i+1}$ is the flow that can be received by the cell $i+1$ from the cell $i ; \varphi_{M, i}$ is the maximum flow allowed by the capacity of cell $i, \rho_{M, i}$ is the jam density (i.e. the maximum density that can be reached), $v_{i}$ corresponds to the free flow speed and $w_{i}$ is the congestion wave speed in cell $i$. This continuous-time description is suitable for the comparison with the variable-length model presented next, and generality of the analysis is not lost.

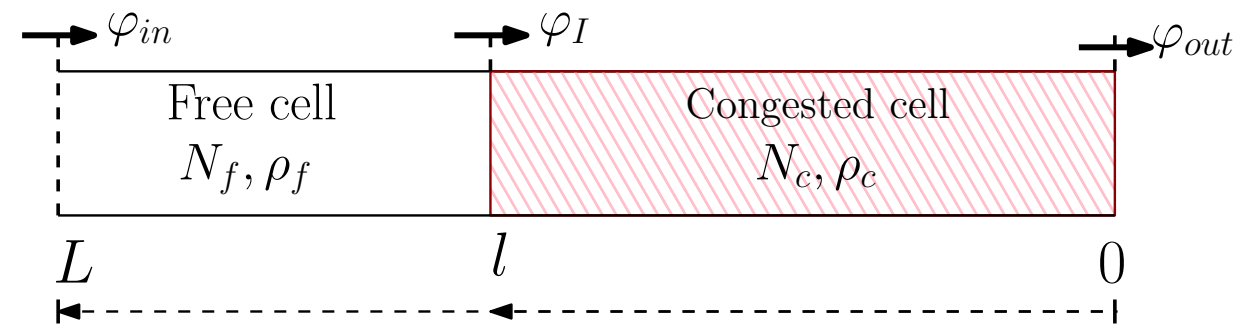

Fig. 2. Schematic diagram of the two-cell variable-length model VLM consisting of three lumped state variables: $\rho_{f}$, $\rho_{c}$ and $l . \varphi_{I}$ is the interface flow between the two cells.

\section{NEW MACROSCOPIC MODEL WITH VARIABLE-LENGTH CELLS}

Consider first a simple road section described by two cells of variable length: a downstream congested cell, of length $l$, and an upstream free cell, of length $L-l$, where $L$ is the total length of the section. The former cell is characterized by the lumped (or averaged) "congested density" $\rho_{c}$, the latter by the lumped (or averaged) "free density" $\rho_{f}$. Note that $l=l(t)$ is timevarying and specifies the position of the congestion wave front, see Fig. 2. Then, in contrast to the $n$-fixed cells model (3), the new macroscopic traffic model introduced in this paper, named Variable-Length cell Model (VLM), consists only of three lumped state variables, i.e. $\rho_{f}, \rho_{c}$ and $l$. Its derivation is done under the assumption that the fundamental diagram is described by the bilinear triangular form shown in Fig. 1.

In the proposed model, the interface flow between the free and the congested cells, denoted as $\varphi_{I}$, plays a central role. Indeed, in this section we will present the model derivation in two steps. 
First, we will write the conservation equation adapted to our specific case of variable-length cells as a function of $\varphi_{I}$, and the variation law of $l$. Due to the time-varying nature of cells length, $\varphi_{I}$ cannot be computed as in the fixed-length cell case by (4), but needs to be redefined using a relative velocity frame to preserve consistency with the evolution in time of the congestion front. This will be performed in the second step of the model derivation.

Remark 1 Note that the model presents three degenerate cases when $l=0, l=L$, and $\rho_{f}=\rho_{c}$. Those cases concern the situations in which the constituent separation assumption between the free and congested modes is violated. The treatment of such cases will be addressed at the end of the section.

\section{A. Conservation law for the variable-length cells}

Consider, for consistency with the grow direction of $l$ shown in Fig. 2, that the $x$-axis is oriented with $l$ from right (downstream) to left (upstream). Therefore, the conservation law (2) now can be written as $\partial_{t} \rho=\partial_{x} \Phi(\rho)$, where we have changed $\partial_{x}$ by $-\partial_{x}$. Moreover, let $s(t)$ be the continuous variable describing the time-varying shock wave position along the $x$ axis. Relying on $s(t)$, the following lumped (averaged) densities variables $\rho_{f}$ and $\rho_{c}$ are defined:

$$
\begin{aligned}
\rho_{f} & =\frac{1}{L-s} \int_{s}^{L} \rho(x, t) d x \\
\rho_{c} & =\frac{1}{s} \int_{0}^{s} \rho(x, t) d x
\end{aligned}
$$

Note that the density distribution is discontinuous at the boundary $x=s$. Its left, $\rho^{-}$, and right, $\rho^{+}$, limits can be written as

$$
\rho^{-}=\rho^{-}(t)=\lim _{x \rightarrow s^{-}} \rho(x, t), \quad \text { and } \quad \rho^{+}=\rho^{+}(t)=\lim _{x \rightarrow s^{+}} \rho(x, t)
$$

The time-derivatives of the lumped variables are given by

$$
\begin{aligned}
\dot{\rho}_{f} & =\frac{1}{L-s}\left[\int_{s}^{L} \partial_{t} \rho(x, t) d x-\rho^{-} \dot{s}\right]+\frac{\dot{s}}{(L-s)^{2}} \int_{s}^{L} \rho(x, t) d x \\
\dot{\rho}_{c} & =\frac{1}{s}\left[\int_{0}^{s} \partial_{t} \rho(x, t) d x+\rho^{+} \dot{s}\right]-\frac{\dot{s}}{s^{2}} \int_{0}^{s} \rho(x, t) d x
\end{aligned}
$$

Using $\partial_{t} \rho(x, t)=\partial_{x} \Phi(\rho)$ in the previous integrals, and the definitions (5a)-(5b), $\dot{\rho}_{f}$ rewrites as

$$
\begin{aligned}
\dot{\rho}_{f} & =\frac{1}{L-s}\left[\Phi(\rho(L))-\Phi\left(\rho^{-}\right)-\rho^{-} \dot{s}\right]+\frac{\dot{s}}{(L-s)} \rho_{f} \\
& =\frac{1}{L-s}\left[\varphi_{i n}-\Phi\left(\rho^{-}\right)-\rho^{-} \dot{s}\right]+\frac{\dot{s}}{(L-s)} \rho_{f}
\end{aligned}
$$


whith the boundary upstream flow $\Phi(\rho(L))=\varphi_{i n}$. Similarly, we get for $\dot{\rho}_{c}$

$$
\begin{aligned}
\dot{\rho}_{c} & =\frac{1}{s}\left[\Phi\left(\rho^{+}\right)-\Phi(\rho(0))+\rho^{+} \dot{s}\right]-\frac{\dot{s}}{s} \rho_{c} \\
& =\frac{1}{s}\left[\Phi\left(\rho^{+}\right)-\varphi_{\text {out }}+\rho^{+} \dot{s}\right]-\frac{\dot{s}}{s} \rho_{c}
\end{aligned}
$$

where $\Phi(\rho(0))=\varphi_{\text {out }}$, and the boundary densities and flows at the shock wave front are denoted as $\rho^{-}, \rho^{+}$, and $\Phi\left(\rho^{+}\right), \Phi\left(\rho^{-}\right)$, respectively. From the Rankine-Hugoniot jump condition, the shock wave speed, $\dot{s}$ can be written, in the $l$ coordinates, as

$$
\dot{s}=\frac{\Phi\left(\rho^{-}\right)-\Phi\left(\rho^{+}\right)}{\rho^{+}-\rho^{-}}
$$

implying that $\Phi\left(\rho^{-}\right)+\rho^{-} \dot{s}=\Phi\left(\rho^{+}\right)+\rho^{+} \dot{s}$. The balance between the first term (left-flow) and the second term (out-flow) is a direct consequence of the principle of vehicle conservation at the interface. The "moving" interface flow $\varphi_{I}$ is given by

$$
\varphi_{I}=\Phi\left(\rho^{+}\right)+\rho^{+} \dot{s}=\Phi\left(\rho^{-}\right)+\rho^{-} \dot{s}
$$

Therefore, equations (8) and (9) can be rewitten as

$$
\begin{aligned}
\dot{\rho}_{f} & =\frac{1}{L-s}\left[\varphi_{\text {in }}-\varphi_{I}+\dot{s} \rho_{f}\right] \\
\dot{\rho}_{c} & =\frac{1}{s}\left[\varphi_{I}-\varphi_{\text {out }}-\dot{s} \rho_{c}\right]
\end{aligned}
$$

Note that all the variables in the previous equations, except for $s$, are expressed in the lumped form. In order to arrive at a description which only depends on lumped variables, an approximation of the shock wave position $s(t)$, denoted with $l(t)$, is introduced. The time evolution of $l(t) \in(0, L)$ is proposed to be

$$
i=\frac{\Phi\left(\rho_{f}\right)-\Phi\left(\rho_{c}\right)}{\rho_{c}-\rho_{f}}
$$

which can be regarded as the lumped version of (10). More specifically, in (14) we assume that $\Phi\left(\rho^{-}\right)$and $\Phi\left(\rho^{+}\right)$are approximated by $\Phi\left(\rho_{f}\right)$ and $\Phi\left(\rho_{c}\right)$, respectively. This approximation makes sense as the densities in each of the variable cells are assumed to be scalar and constant over the space variables, and not distributions.

Then on the basis of (14), equations (12) and (13) can be rewritten as

$$
\begin{aligned}
\dot{\rho}_{f} & =\frac{1}{L-l}\left[\varphi_{\text {in }}-\varphi_{I}+i \rho_{f}\right] \\
\dot{\rho}_{c} & =\frac{1}{l}\left[\varphi_{I}-\varphi_{\text {out }}-i \rho_{c}\right]
\end{aligned}
$$


Remark 2 Note that the same type of equations can be obtained by considering the number of vehicles in the free part, $N_{f}=(L-l) \rho_{f}$, and in the congested part, $N_{c}=l \rho_{c}$, and applying the conservation law $\dot{N}=\varphi_{\text {in }}-\varphi_{\text {out }}$ to each of the two parts, i.e.

$$
\begin{aligned}
& \dot{N}_{f}=\varphi_{\text {in }}-\varphi_{I} \\
& \dot{N}_{c}=\varphi_{I}-\varphi_{\text {out }}
\end{aligned}
$$

Substitution of the definition of $N_{f}$ and $N_{c}$ in these equations leads to (12) and (13). The derivation of these equations presented in this paper explicitly formalizes the interpretation of the "lumped" variables as being the average values of the distributed densities in the respective cells.

\section{B. Full dynamics}

Note that the lumped model provides a piece-wise value of the traffic density for each of the two portions (the free and the congested one) of the road section, in opposition to the spatially distributed formulation. The boundary density values in the lumped case are then given as

$$
\rho^{+}=\rho_{c}, \quad \text { and } \quad \rho^{-}=\rho_{f}
$$

so that equation (11) writes now as

$$
\varphi_{I}=\Phi\left(\rho_{c}\right)+\rho_{c} \dot{l}=\Phi\left(\rho_{f}\right)+\rho_{f} \dot{l}
$$

from which we get

$$
-\varphi_{I}+\rho_{f} i=-\Phi\left(\rho_{f}\right) \quad \text { and } \quad \varphi_{I}-\rho_{c} i=\Phi\left(\rho_{c}\right)
$$

Substitution of these expression into (15) and (16), together with (14), gives

$$
\begin{aligned}
\dot{\rho}_{f} & =\frac{1}{L-l}\left[\varphi_{\text {in }}-\Phi\left(\rho_{f}\right)\right] \\
\dot{\rho}_{c} & =\frac{1}{l}\left[\Phi\left(\rho_{c}\right)-\varphi_{\text {out }}\right] \\
i & =\frac{\Phi\left(\rho_{f}\right)-\Phi\left(\rho_{c}\right)}{\rho_{c}-\rho_{f}}
\end{aligned}
$$

Equations (21)-(23) define the new macroscopic traffic model we propose. This model is a lumped variables model based on three state variables only. As for its structure, it is simpler than that of classical continuous macroscopic models and $n$-fixed cells models. Nevertheless, 
as discussed in the next section, it features interesting properties which allow it to capture the relevant phenomena of traffic dynamics.

Remark 3 Equations (21)-(22) describe a non-linear set of ordinary differential equations with "state-dependent" convergence proprieties induced by the terms $1 /(L-l)$ in (21), and $1 / l$ in (22), respectively. To efficiently solve these equations it is then advised to use variable-step ODEs solvers (i.e. ode45, ode23). Note that at the exact boundaries when $l=0$ or to $l=L$, the Equations (21)-(22) formally become a differential algebraic equations. To prevent this situation we add (see next section) further features to the model to limit the evolution of $l$ to the set $l \in[\epsilon, L-\epsilon]$, and thus be able to solve the equation with simpler ODE solvers. It is also worth to observe that when $l=\epsilon$ and $l=L-\epsilon$ equations (21)-(22) will exhibit a "stiff" behavior displaying an important time-scale separation between the slow and the fast modes. Using ODEs solvers $^{1}$ for stiff equations are then highly recommended.

\section{MATHEMATICAL PROPERTIES}

In this section we present some of the main mathematical properties of the proposed model with the bilinear triangular form for the fundamental diagram as shown in Fig. 1. We first show that the model provides time-solutions consistent with the constructive assumptions (i.e. states remain in their definition domain). This property is named "consistency" and can also be understood as an invariant property. Then we analyze equilibrium points and their associated stability. Finally, we treat the singular points where $l$ is equal to either 0 or $L$.

\section{A. Model consistency}

Let first define the following sets

$$
\begin{aligned}
& \Omega_{f}=\left\{\rho: \rho \in\left[0, \rho^{*}\right]\right\} \\
& \Omega_{c}=\left\{\rho: \rho \in\left[\rho^{*}, \rho_{M}\right]\right\} \\
& \Omega_{l}=\{l: l \in(0, L)\}
\end{aligned}
$$

Property 1 (Density invariance) Assume that $l \in \Omega_{l}$ for all $t \geq 0$, then the following holds:

\footnotetext{
${ }^{1}$ Examples of ODEs solvers for stiff equations are: Backward differentiation formulae, Gear methods, LSODE, VODE, etc. Some of those are freely distributed evolving into ODEPACK or Sundials packages, while other are options in the MatLab sotfware
} 
i) If $\rho_{f}(0) \in \Omega_{f}$, then $\rho_{f}(t) \in \Omega_{f}, \forall t \geq 0$

ii) If $\rho_{c}(0) \in \Omega_{c}$, then $\rho_{c}(t) \in \Omega_{c}, \forall t \geq 0$

along the time-solutions of the equation set (21)-(22)

Proof. The proof follows by inspecting the time-derivative of $\rho_{f}$ and $\rho_{c}$ at the closure of their respective sets.

Consider first the dynamics of $\rho_{f}$. Property $i$ ) is equivalent to

$$
\begin{aligned}
& \dot{\rho}_{f} \geq 0 \quad \text { at } \quad \rho_{f}=0 \\
& \dot{\rho}_{f} \leq 0 \quad \text { at } \quad \rho_{f}=\rho^{*}
\end{aligned}
$$

Note that $0 \leq \varphi_{\text {in }} \leq \varphi_{M}=v \rho^{*}$, and $\Phi\left(\rho_{f}\right)=v \rho_{f}$. For the first case $\left(\rho_{f}=0\right)$, we have:

$$
\dot{\rho}_{f}=\frac{1}{L-l} \varphi_{i n} \geq 0
$$

while, for the second case $\left(\rho_{f}=\rho^{*}\right)$, we have

$$
\dot{\rho}_{f}=\frac{1}{L-l}\left[\varphi_{i n}-v \rho^{*}\right] \leq 0
$$

which follows by virtue of the property $\varphi_{\text {in }} \leq v \rho^{*}$. Consider now the dynamics of $\rho_{c}$. Property ii) is equivalent to

$$
\begin{aligned}
& \dot{\rho}_{c} \geq 0 \quad \text { at } \quad \rho_{f}=\rho^{*} \\
& \dot{\rho}_{c} \leq 0 \quad \text { at } \quad \rho_{f}=\rho_{M}
\end{aligned}
$$

note that $0 \leq \varphi_{\text {out }} \leq \varphi_{M}=-w\left(\rho^{*}-\rho_{M}\right)$, and $\Phi\left(\rho_{c}\right)=-w\left(\rho_{c}-\rho_{M}\right)$. For $\rho_{c}=\rho^{*}$, we have

$$
\dot{\rho}_{c}=\frac{1}{l}\left[-w\left(\rho^{*}-\rho_{M}\right)-\varphi_{\text {out }}\right] \geq 0
$$

while at $\rho_{c}=\rho_{M}$, we have

$$
\dot{\rho}_{c}=-\frac{1}{l} \varphi_{\text {out }} \leq 0
$$

which concludes the proof.

\section{B. Equilibrium points}

We now study the equilibrium points of the proposed lumped model and compare them, for consistency, to the equilibrium points of the LWR distributed model.

Property 2 (Equilibrium points) Assume that the inflow and the outflow are constant and given by $\varphi_{\text {in }}=\varphi_{\text {out }}=\bar{\varphi}$, then 
i) The equilibrium points for the densities $\left(\bar{\rho}_{f}, \bar{\rho}_{c}\right)$ are:

$$
\bar{\rho}_{f}=\bar{\varphi} / v, \quad \bar{\rho}_{c}=\rho_{M}-\bar{\varphi} / w
$$

ii) The equilibrium set $\left(\bar{\rho}_{f}, \bar{\rho}_{c}\right)$ belongs to the manifold: $v \bar{\rho}_{f}+w \bar{\rho}_{c}=w \rho_{M}$

iii) Let $\bar{\rho}_{f}<\bar{\rho}_{c}$. The admissible equilibrium set for $l$ and $N$ is:

$$
\Omega_{\bar{l}, \bar{N}}=\left\{(\bar{l}, \bar{N}): \bar{l} \in \Omega_{l}, \bar{N}>0, \bar{l}=\frac{\bar{N}-\bar{\rho}_{f} L}{\bar{\rho}_{c}-\bar{\rho}_{f}}\right\}
$$

where $\bar{N}$ is the number of vehicles in the section at the equilibrium.

Proof. Property $i$ ) directly follows from inspection of equations (21)-(22), by noticing that equilibrium is only possible when $\varphi_{\text {in }}=\varphi_{\text {out }}=\bar{\varphi}$, which implies that $\Phi\left(\bar{\rho}_{f}\right)=\Phi\left(\bar{\rho}_{c}\right)=\bar{\varphi}$. Property $i i$ ) is obtained by eliminating $\bar{\varphi}$ from the equilibrium equations (31). The admissible set as defined in $i i i$ ) derives from the admissible set of $l$, the positive-definitiveness of $N$, and from the vehicle conservation property

$$
N(t)=\rho_{f}(t)(L-l(t))+\rho_{c}(t) l(t)
$$

at the equilibrium.

Remark 4 Note that, under the assumption $\varphi_{\text {in }}=\varphi_{\text {out }}=\bar{\varphi}$, the equilibrium of the lumped model, is also an equilibrium of the LWR equation (2) if the initial distribution $\rho^{0}(x)$ in the LWR model is taken piece-wise constant with values given by (31), with a congested part length given by (32).

Because (23) is undefined at the equilibrium, the equilibria point of this equations are derived from the conservation property of the model. In that, the following study of equilibrium points resumes to look at the stability of the density evolution around their equilibrium points, and then analyze the asymptotic values of $l$ at those points.

\section{Stability of equilibrium points}

As for the stability of the VLM, the following result can be proved.

Theorem 1 (Equilibrium points stability) Let $\varphi_{\text {in }}=\varphi_{\text {out }}=\bar{\varphi}$. Assume also that $l(t) \in \Omega_{l}$. Consider the equilibrium density values in (31), and any couple $(\bar{l}, \bar{N}) \in \Omega_{\bar{l}, \bar{N}}$, then the following holds:

i) $\lim _{t \rightarrow \infty} \rho_{f}(t)=\bar{\rho}_{f}$, exponentially fast with a rate $e^{\frac{-v t}{L}}$ 
ii) $\lim _{t \rightarrow \infty} \rho_{c}(t)=\bar{\rho}_{c}$, exponentially fast with a rate $e^{\frac{-w t}{L}}$

iii) $\lim _{t \rightarrow \infty} l(t)=\bar{l}$, exponentially fast with a rate $e^{\frac{-w t}{L}}$

Proof. Let introduce the error variables: $\tilde{\rho}_{f}=\rho_{f}-\bar{\rho}_{f}, \tilde{\rho}_{c}=\rho_{c}-\bar{\rho}_{c}$. The error dynamics along the solutions of (21)-(22) can be easily computed as

$$
\begin{aligned}
& \dot{\tilde{\rho}}_{f}=-\frac{v}{L-l} \tilde{\rho}_{f} \leq-\frac{v}{L} \tilde{\rho}_{f} \\
& \dot{\tilde{\rho}}_{c}=-\frac{w}{l} \tilde{\rho}_{c} \leq-\frac{w}{L} \tilde{\rho}_{c}
\end{aligned}
$$

where the inequalities derive from the fact that $l(t) \in \Omega_{l}$. From here we have that $\tilde{\rho}_{f}(t) \leq$ $\tilde{\rho}_{f}(0) e^{\frac{-v t}{L}}$, and $\tilde{\rho}_{c}(t) \leq \tilde{\rho}_{c}(0) e^{\frac{-w t}{L}}$. Evaluation of the conservation equation (33) at its limit, for $t \rightarrow \infty$, gives

$$
N(\infty)=\rho_{f}(\infty)(L-l(\infty))+\rho_{c}(\infty) l(\infty)
$$

Under the assumption $\varphi_{\text {in }}=\varphi_{\text {out }}=\bar{\varphi}$, we have that $\dot{N}=0$, hence $N(t)$ is constant and $N(t)=N(\infty)=\bar{N}$. Due to the exponential stability of the densities, we have that $\rho_{f}(\infty)=\bar{\rho}_{f}$, and $\rho_{c}(\infty)=\bar{\rho}_{c}$. The previous equation exponentially converge to

$$
\bar{N}=\bar{\rho}_{f}(L-l(\infty))+\bar{\rho}_{c} l(\infty)
$$

with a rate given by $\min \left\{e^{\frac{-v t}{L}}, e^{\frac{-w t}{L}}\right\}=e^{\frac{-w t}{L}}$. This gives,

$$
l(\infty)=\frac{\bar{N}-\bar{\rho}_{f} L}{\bar{\rho}_{c}-\bar{\rho}_{f}}=\bar{l}
$$

which proves $i i i)$.

\section{Treatment of singular points}

Model (21)-(22), can also be rewritten as:

$$
\begin{aligned}
(L-l) \dot{\rho}_{f} & =\varphi_{\text {in }}-\Phi\left(\rho_{f}\right) \\
l \dot{\rho}_{c} & =\Phi\left(\rho_{c}\right)-\varphi_{\text {out }} \\
\left(\rho_{c}-\rho_{f}\right) \dot{l} & =\Phi\left(\rho_{f}\right)-\Phi\left(\rho_{c}\right)
\end{aligned}
$$

describing a set of non-linear ordinary differential equations (ODEs). However, at the three degenerate cases when $l=0, l=L$, and $\rho_{f}=\rho_{c}$, the model formally become a differential algebraic equations (DAEs) as at the singularities the time-differential equation transforms to an 
algebraic relation. It is still possible to treat the model as a DAE, at the price of a complexity increase in terms of analysis and numerical solutions. Instead, it is possible (as we propose in this section), to modify the model to cope with this singularities while preserving the continuity and the typology of the solutions. With these modifications the model can still be solved with standard ODEs solvers.

In this subsection we shown how the original model can be reformulated to treat such singular cases. We first present the solution for the cases when $l=0, l=L$, and show that the proposed modifications preserve the time-continuity of the solutions for $\rho$. Then, we present the treatment of the second class of singularity arriving when $\rho_{f}=\rho_{c}$, and show that the regularization also preserve continuity of the solution of $l$.

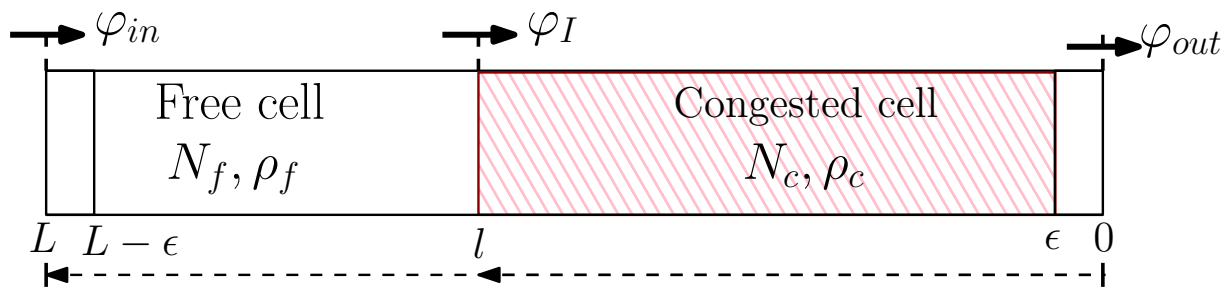

Fig. 3. Modification of the original VLM with two small constant cells at the boundaries introduced to avoid singularities at $l=0$, and $l=L$.

1) Singular points for $l(t)$ : Consider first the case where the variable length $l(t)$ approaches one of the two possible boundary values. In those cases, the idea is to create a $\varepsilon$-boundary layer at the two extremes of the considered road section and to stop the evolution of $l(t)$ when it reaches the $\varepsilon$-boundary layer borders. When this happens, the model collapses into the standard CTM model with fixed length cells, and the density variables evolve according to the conventional "Demand-Supply" mechanism, with the standard notation

$$
D(\rho)=\min \left\{v \rho, \varphi_{M}\right\}, \quad S(\rho)=\min \left\{-w\left(\rho-\rho_{M}\right), \varphi_{M}\right\}
$$

This idea is sketched in Fig. 3.

Let us consider an arbitrarily small $\varepsilon$ such that $0<\varepsilon \ll L$. Then the following two conditions can be introduced:

$$
\begin{aligned}
\mathcal{C}_{0} & =\left\{(l=\varepsilon) \cap\left(D\left(\rho_{f}\right) \leq S\left(\rho_{c}\right)\right)\right\} \\
\mathcal{C}_{L} & =\left\{(l=L-\varepsilon) \cap\left(D\left(\rho_{f}\right) \geq S\left(\rho_{c}\right)\right)\right\}
\end{aligned}
$$


Condition $\mathcal{C}_{0}$ implies that the lower extreme is reached while the congestion front wave is moving downstream. Symmetrically, condition $\mathcal{C}_{L}$ indicates that the upper extreme is reached while the congestion front wave is moving upstream. For those specific cases, the following model modification is proposed:

- If $\mathcal{C}_{0}$ holds, the interface flow is $\varphi_{I}=\min \left\{D\left(\rho_{f}\right), S\left(\rho_{c}\right)\right\}=D\left(\rho_{f}\right)$, and then the model becomes

$$
\begin{aligned}
\dot{\rho}_{f} & =\frac{1}{L-\varepsilon}\left[\varphi_{\text {in }}-D\left(\rho_{f}\right)\right] \\
\dot{\rho}_{c} & =\frac{1}{\varepsilon}\left[D\left(\rho_{f}\right)-\varphi_{\text {out }}\right] \\
i & =0
\end{aligned}
$$

Note that in this case, the variation domain of the state variables is partially modified. The congested density variable can now take values smaller than the critical density $\rho^{*}$, i.e. $\rho_{c} \in\left[0, \rho_{M}\right]$, while the free density variable remains in its original domain, i.e. $\rho_{f} \in \Omega_{f}$.

- If $\mathcal{C}_{L}$ holds, the interface flow is $\varphi_{I}=\min \left\{D\left(\rho_{f}\right), S\left(\rho_{c}\right)\right\}=S\left(\rho_{c}\right)$, and then the model becomes

$$
\begin{aligned}
\dot{\rho}_{f} & =\frac{1}{\varepsilon}\left[\varphi_{\text {in }}-S\left(\rho_{c}\right)\right] \\
\dot{\rho}_{c} & =\frac{1}{L-\varepsilon}\left[S\left(\rho_{c}\right)-\varphi_{\text {out }}\right] \\
\dot{l} & =0
\end{aligned}
$$

In this case, the free density variable can take values larger than the critical density $\rho^{*}$, i.e. $\rho_{f} \in\left[0, \rho_{M}\right]$, while the congested density variable remains in its original domain, i.e. $\rho_{c} \in \Omega_{c}$.

Property 3 (Continuity of the solution at the transitions) The time-evolution of the solutions when the model switches from regular operation to/from operation under conditions $\mathcal{C}_{0}$ or $\mathcal{C}_{L}$ is continuous in time.

Proof. Consider the case of condition $\mathcal{C}_{0}$. Equation (38) remains the same when the transition occurs at $l=\varepsilon$. The right-hand side of (39) undergoes a discontinuity due to the transition $S\left(\rho_{c}\right) \rightarrow D\left(\rho_{f}\right)$. This can be quantified as follows

$$
\begin{aligned}
\dot{\rho}_{c} & =\frac{1}{\varepsilon}\left[S\left(\rho_{c}\right)-\varphi_{\text {out }}\right]+\frac{1}{\varepsilon}\left[D\left(\rho_{f}\right)-S\left(\rho_{c}\right)\right] \\
& =\frac{1}{\varepsilon}\left[S\left(\rho_{c}\right)-\varphi_{\text {out }}\right]+\delta\left(\rho_{f}, \rho_{c}\right)
\end{aligned}
$$


where $\delta\left(\rho_{f}, \rho_{c}\right)$ captures the transition effect. It can be easily shown that $\delta\left(\rho_{f}, \rho_{c}\right)$ is bounded as: $\left|\delta\left(\rho_{f}, \rho_{c}\right)\right| \leq \varphi_{M} / \varepsilon$, and therefore its contribution to the solution $\rho_{c}(t)$ (namely $\Delta \rho_{c}(t)$ ) is also bounded as

$$
\left|\Delta \rho_{c}(t)\right| \leq \frac{\varphi_{M}}{\varepsilon}\left(t-t_{T}\right)
$$

where $t_{T}$ is the time instant at which the transition occurs. This implies the continuity of the solution at the transition time instant. The same type of analysis can be carried out for the solutions of equation (40).

Similar considerations are due for the case of $\mathcal{C}_{L}$, with the exception that in this case equation (42) remains the same when the transition occurs at $l=L-\varepsilon$, whereas the right-hand side of (41) undergoes a discontinuity, now due to the transition $D\left(\rho_{f}\right) \rightarrow S\left(\rho_{c}\right)$.

2) Singular points at $\rho_{f}=\rho_{c}$ : Now consider the third singular point, i.e. $\rho_{f}=\rho_{c}$. From the fundamental diagram in Fig. 1, it is apparent that this singularity only occurs at $\rho_{f}=\rho_{c}=$ $\rho^{*}$, where $\Phi\left(\rho_{f}\right)=\Phi\left(\rho_{c}\right)=\varphi_{M}$. This implies that equation (23) becomes undetermined. As a consequence, in order to extend the proposed model validity to this case, a regularization mechanism needs to be introduced. To this end, let $e_{f}=\rho_{f}-\rho^{*} \leq 0$ and $e_{c}=\rho_{c}-\rho^{*} \geq 0$, we have that $e_{f}=-\left|e_{f}\right|$, and $e_{c}=\left|e_{c}\right|$. Hence equation (23) can be rewritten as,

$$
i=\frac{-v\left|e_{f}\right|+w\left|e_{c}\right|}{\left|e_{f}\right|+\left|e_{c}\right|}
$$

Let us denote with $V\left(e_{f}, e_{c}\right)=\left|e_{f}\right|+\left|e_{c}\right|$, and with $\sigma\left(e_{f}, e_{c}\right)=\epsilon e^{-\alpha V^{2}\left(e_{f}, e_{c}\right)}, \epsilon$ and $\alpha$ being strictly positive coefficients, a suitable regularizing term. Then, the following regularization for (23)

$$
i=\frac{-v\left|e_{f}\right|+w\left|e_{c}\right|}{V\left(e_{f}, e_{c}\right)+\sigma\left(e_{f}, e_{c}\right)}, \quad \lim _{V\left(e_{f}, e_{c}\right) \rightarrow 0} i=0
$$

is proposed, which in the original system coordinates writes as

$$
i=\frac{\Phi\left(\rho_{f}\right)-\Phi\left(\rho_{c}\right)}{\rho_{c}-\rho_{f}+\sigma\left(\rho_{c}, \rho_{f}\right)}
$$

with $\sigma\left(\rho_{c}, \rho_{f}\right)=\epsilon e^{-\alpha\left(\rho_{f}-\rho_{c}\right)^{2}}$. 


\section{E. Singularity-free full VLM}

Considering the different regularizations introduced previously, and noticing that $l=\varepsilon$, and $l=L-\varepsilon$, at $\mathcal{C}_{0}$ and $\mathcal{C}_{L}$, respectively, the full model can be compactly written as follows

$$
\begin{gathered}
\dot{\rho}_{f}=\frac{1}{L-l}\left\{\begin{array}{ccc}
\varphi_{\text {in }}-D\left(\rho_{f}\right) & \text { if } & \mathcal{C}_{0} \\
\varphi_{\text {in }}-S\left(\rho_{c}\right) & \text { if } & \mathcal{C}_{L} \\
\varphi_{\text {in }}-\Phi\left(\rho_{f}\right) & \text { else }
\end{array}\right. \\
\dot{\rho}_{c}=\frac{1}{l}\left\{\begin{array}{ccc}
D\left(\rho_{f}\right)-\varphi_{\text {out }} & \text { if } & \mathcal{C}_{0} \\
S\left(\rho_{c}\right)-\varphi_{\text {out }} & \text { if } & \mathcal{C}_{L} \\
\Phi\left(\rho_{c}\right)-\varphi_{\text {out }} & \text { else }
\end{array}\right. \\
i=\left\{\begin{array}{ccc}
0 & \text { if } & \mathcal{C}_{0} \bigcup \mathcal{C}_{L} \\
\frac{\Phi\left(\rho_{f}\right)-\Phi\left(\rho_{c}\right)}{\rho_{c}-\rho_{f}+\sigma\left(\rho_{c}, \rho_{f}\right)} & \text { else }
\end{array}\right.
\end{gathered}
$$

with this model, the definition domains $\left(\Omega_{f}^{\varepsilon}, \Omega_{c}^{\varepsilon}, \Omega_{l}^{\varepsilon}\right)$ for this model are now:

$$
\begin{aligned}
\rho_{f} \in \Omega_{f}^{\varepsilon} & =\left\{\begin{array}{cc}
\Omega_{f} \cup \Omega_{c} & \text { if } \mathcal{C}_{L} \\
\Omega_{f} & \text { else }
\end{array}\right. \\
\rho_{c} \in \Omega_{c}^{\varepsilon} & =\left\{\begin{array}{cc}
\Omega_{f} \cup \Omega_{c} & \text { if } \mathcal{C}_{0} \\
\Omega_{c} & \text { else }
\end{array}\right. \\
l \in \Omega_{l}^{\varepsilon} & =\{l: l \in[\varepsilon, L-\varepsilon]\}
\end{aligned}
$$

and it is straightforward to see that if initial conditions $\left(\rho_{f}(0), \rho_{c}(0), l(0)\right)$ are taken in this domain $\left(\Omega_{f}^{\varepsilon} \times \Omega_{c}^{\varepsilon} \times \Omega_{l}^{\varepsilon}\right)$, and the boundary flows are consistent with this initial conditions, then the solutions of the (45)-(45) will remain in this domain. Note that as mention before, $\rho_{f}$ (respectively $\rho_{c}$ ) can take any value when $\mathcal{C}_{L}$ (respectively $\mathcal{C}_{0}$ ) is active as the model in that modes, behaves as a standard CTM with two cells (one very large, and other very small).

\section{INCLUDING RAREFACTION WAVES IN THE VLM}

Model (45)-(47) has been designed under the implicit assumption of the existence of a bottleneck originating an important change of capacity at the boundary $l=0$, and producing a congestion whose origin in the space remains fixed. This is typically the case when the congestion is originated at some fixed spatial localization (i.e. at a merging/splitting node, by a red traffic light, by a car accident, etc.) and the congestion is built upstream from this point. However, there are other situations in which both spatial boundaries of the congestion may evolve in time. This 
is the case when a suddenly change from congested to free occurs at the downstream boundary (i.e. because of the traffic light switching from red to green, or of the car accident eventually resolved) producing a rarefaction (or fan) wave at the downstream boundary, as shown in Fig. 4.

In this section, we first formalize the conditions for the existence of shock and rarefaction waves for characteristic curves which are piece-wise linear (triangular) functions, and then we propose an extension of model (45)-(47) to include the rarefaction case as well. The overall model results in being a model with a time-varying downstream and upstream boundaries as illustrated in Fig. 4.
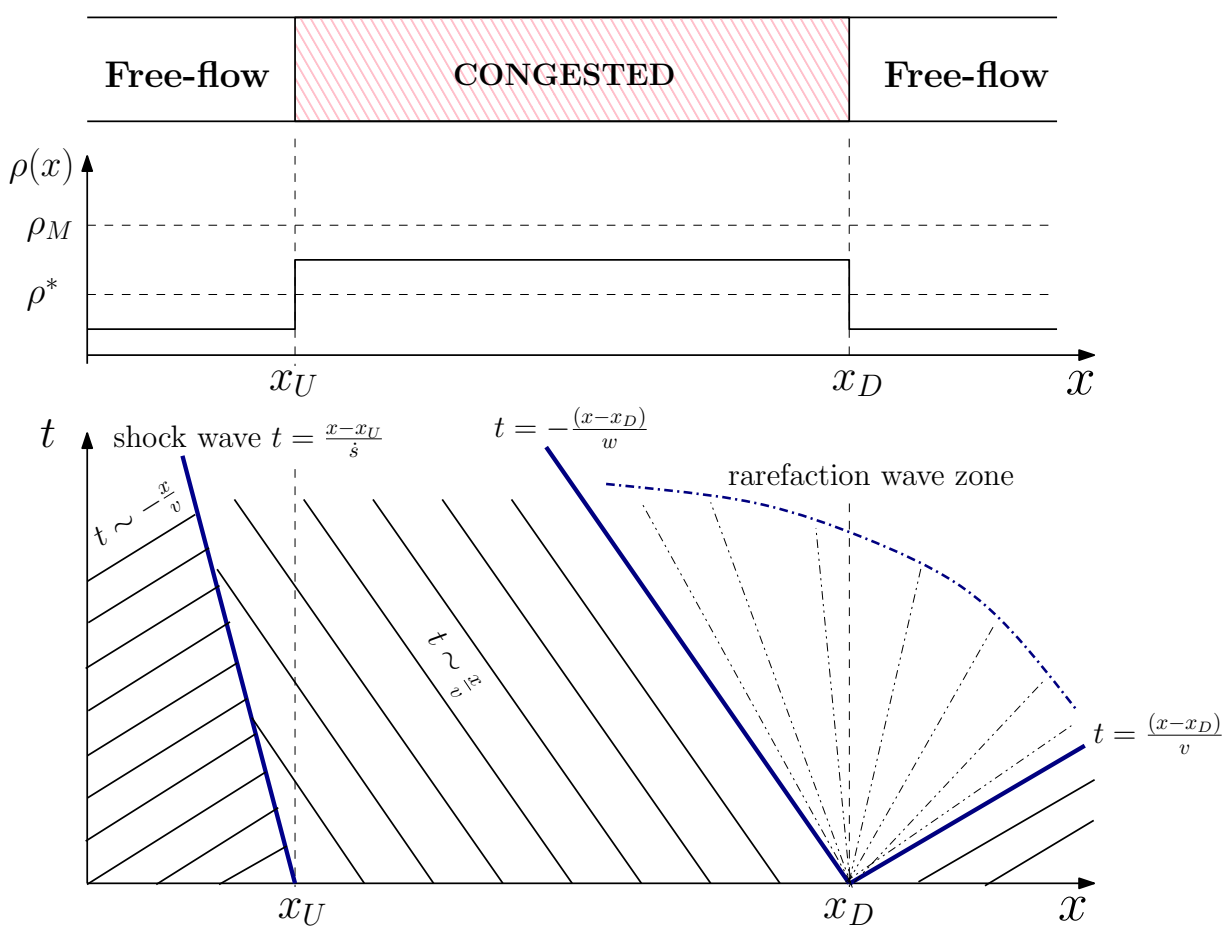

Fig. 4. Illustration of characteristics corresponding to the shock and the rarefaction waves occurring at the upstream and downstream boundaries located at $x_{U}$ and $x_{D}$ respectively. The solutions are continuous and non-unique in the rarefaction wave zone if $\Phi(\rho)$ is continuously differentiable. For those cases, the density takes values in the set $\rho \in\left[\rho^{-}, \rho^{+}\right]$. If $\Phi(\rho)$ is piece-wise triangular, then the method of characteristics does not allow to identify a solution in this region. However, the solutions are now defined in the weak-sense, unique, and given by $\rho=\rho^{*}$ for all $(x, t)$ in the cone $\mathcal{C}\left[t=-\frac{x-x_{D}}{w}, t=\frac{x-x_{D}}{v}\right]$.

\section{A. Shock and rarefaction waves at the boundaries}

Shock waves at the upstream boundary $x_{U}$. Consider the Riemann problem with initial distributions as shown in Fig. 4 with $0<\rho^{-}<\rho^{*}<\rho^{+}<\rho_{M}$ at the upstream boundary $u_{U}$. 
Then a shock exist and the discontinuity will propagate with the speed $\dot{s}_{x}=\frac{\Phi\left(\rho^{-}\right)-\Phi\left(\rho^{+}\right)}{\rho^{-}-\rho^{+}}$in the left-to-right $x$-direction under the following conditions:

a) For continuously differentiable functions $\Phi(\rho)$ the Lax entropy condition:

$$
\Phi^{\prime}\left(\rho^{-}\right)>\dot{s}_{x}>\Phi^{\prime}\left(\rho^{+}\right)
$$

holds, where $\Phi^{\prime}(\rho)=\partial \Phi(\rho) / \partial \rho$. For concave functions $\Phi(\rho)$ the entropy condition is satisfied if $\rho^{-}<\rho^{+}$, and solutions are uniquely defined.

b) For piece-wise continuous functions $\Phi(\rho)$, as the triangular form adopted here, the Lax entropy condition (48) is no longer valid. In that case, the Oleinik entropy condition [40] is more appropriate. The simplest form of this condition, as proposed by Dafermos [13], is given by

$$
\begin{aligned}
& \Phi(\rho) \geq C(\rho), \forall \rho \in\left[\rho^{-}, \rho^{+}\right], \quad \text { if } \quad \rho^{-}<\rho^{+} \\
& \Phi(\rho) \leq C(\rho), \forall \rho \in\left[\rho^{-}, \rho^{+}\right], \quad \text { if } \quad \rho^{-}>\rho^{+}
\end{aligned}
$$

where $C(\rho)=\Phi\left(\rho^{-}\right)+\dot{s}_{x}\left(\rho-\rho^{-}\right)$is the chord between $\rho^{-}$and $\rho^{+}$.

For concave piece-wise triangular functions $\Phi(\rho)$, the chord is always below $\Phi(\rho)$ and thus condition (49) always holds. Hence the (entropy weak) solution always exists and is uniquely defined [23] by

$$
\rho(x, t)= \begin{cases}\rho^{-} & \left(x-x_{U}\right)<\dot{s}_{x} t \\ \rho^{+} & \left(x-x_{U}\right) \geq \dot{s}_{x} t\end{cases}
$$

Rarefaction waves at the downstream boundary $s_{D}$. Consider now the Riemann problem with initial distributions as shown in Fig. 4, but now at the downstream boundary $u_{D}$, where $0<\rho^{+}<\rho^{*}<\rho^{-}<\rho_{M}$.

c) Let $\Phi(\rho)$ be a continuously differentiable function. It can be easily verified that for $\Phi(\rho)$ concave, the entropy condition (48) is never verified, and hence shocks cannot formally exist. Instead a rarefaction wave solution will appear at the downstream boundary, whose general form is given by,

$$
\rho(x, t)=\left\{\begin{array}{lr}
\rho^{-} & \left(x-x_{D}\right)<\Phi^{\prime}\left(\rho^{-}\right) t, \\
\Phi^{\prime}\left(\frac{x}{t}\right)^{-1} & \Phi^{\prime}\left(\rho^{-}\right) t \leq\left(x-x_{D}\right)<\Phi^{\prime}\left(\rho^{+}\right) t, \\
\rho^{+} & \left(x-x_{D}\right) \geq \Phi^{\prime}\left(\rho^{+}\right) t .
\end{array}\right.
$$


giving rise to multiple continuous solutions with density values in the set $\Phi^{\prime}\left(\frac{x}{t}\right)^{-1} \in\left[\rho^{-}, \rho^{+}\right]$, as shown through the characteristics locus (doted-lines) in Fig. $4 . \Phi^{\prime}(\cdot)^{-1}$ is the inverse map of the characteristics $\Phi^{\prime}(\rho)=\frac{x}{t}$.

d) Let $\Phi(\rho)$ be a piece-wise triangular function. Then we can see that the Oleinik entropy condition (50) does not hold for an original distribution of form (66) as the chord $C(q)$ will never be above $\Phi(\rho)$. However, as shown in [23], there exist an unique entropy solution, if one considers the intermediate value of density $\rho^{*}$. In that case, the two discontinuities $\rho^{-} \mid \rho^{*}$, and $\rho^{*} \mid \rho^{+}$satisfy the entropy condition (50) as an equality, and give raise to the following solution

$$
\rho(x, t)=\left\{\begin{array}{lr}
\rho^{-} & \left(x-x_{D}\right)<\Phi^{\prime}\left(\rho^{-}\right) t, \\
\rho^{*} & \Phi^{\prime}\left(\rho^{-}\right) t \leq\left(x-x_{D}\right)<\Phi^{\prime}\left(\rho^{+}\right) t, \\
\rho^{+} & \left(x-x_{D}\right) \geq \Phi^{\prime}\left(\rho^{+}\right) t .
\end{array}\right.
$$

For the triangular piece-wise linear fundamental diagram, we have $\Phi^{\prime}\left(\rho^{-}\right)=-w$, and $\Phi^{\prime}\left(\rho^{+}\right)=v$. Then, we have the formation of two shock waves: one traveling at velocity $-w$, and another traveling at velocity $v$, with critical density as the intermediate state.

B. Model including rarefactions waves

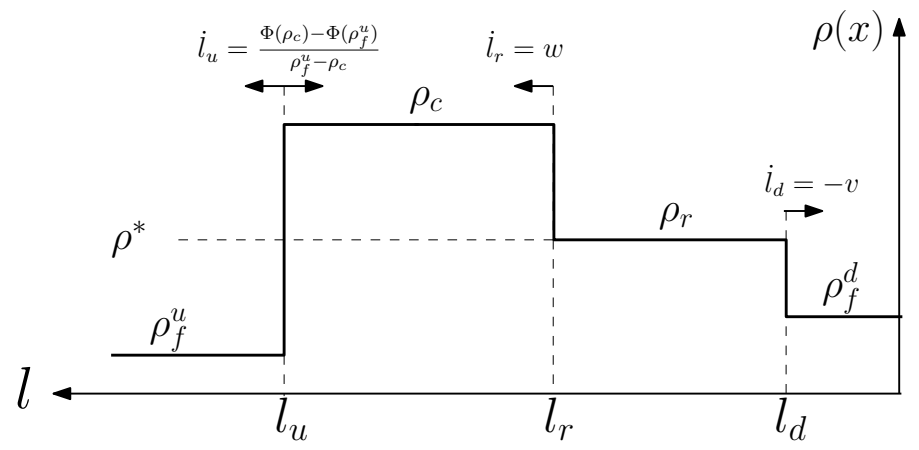

Fig. 5. Illustration of the variables involved in the model including rarefaction waves.

Consider the variables as defined in Fig. 5, with $\rho_{f}^{u}, \rho_{c}, \rho_{r}, \rho_{f}^{d}$ being the densities, and $l_{u}, l_{r}, l_{d}$ being the associated cell lengths subject to the following constraints: $L>l_{u} \geq l_{r} \geq l_{d}>0$. The left boundary of the rarefaction wave is indicated by $l_{r}$, with density $\rho_{r}=\rho^{*}$, as indicated by (51). 
The model can now be constructed by following the same ideas on vehicles conservation laws used in previous sections,

$$
\begin{aligned}
& \dot{N}_{f}^{u}=\varphi_{\text {in }}-\varphi_{I}^{u} \\
& \dot{N}_{c}=\varphi_{I}^{u}-\varphi_{I}^{r} \\
& \dot{N}_{r}=\varphi_{I}^{r}-\varphi_{I}^{d} \\
& \dot{N}_{f}^{d}=\varphi_{I}^{d}-\varphi_{\text {out }}
\end{aligned}
$$

with $N_{f}^{u}=\left(L-l_{u}\right) \rho_{f}^{u}, N_{c}=\left(l_{u}-l_{r}\right) \rho_{c}, N_{r}=\left(l_{r}-L_{d}\right) \rho_{r}, N_{f}^{d}=l_{d} \rho_{f}^{d}$, and $\varphi_{I}^{u}, \varphi_{I}^{r}, \varphi_{I}^{d}$ being the interface flows at $l_{u}, l_{r}, l_{d}$, respectively. As before, each interface flow can be computed using the speed function $i_{-/+}=f\left(\rho^{-}, \rho^{+}\right)=\frac{\Phi\left(\rho^{+}\right)-\Phi\left(\rho^{-}\right)}{\rho^{-}-\rho^{+}}$of each interface, and its respective left and right densities $\rho^{-}, \rho^{+}$. This gives: $\varphi_{I}^{-/+}=\rho^{-} i_{-/+}+\Phi\left(\rho^{-}\right)=\rho^{+} i_{-/+}+\Phi\left(\rho^{+}\right)$. The full model is now given by

$$
\begin{aligned}
& \dot{\rho}_{f}^{u}=\frac{1}{L-l_{u}}\left[\varphi_{\text {in }}-\Phi\left(\rho_{f}^{u}\right)\right] \\
& \dot{\rho}_{c}=0 \\
& \dot{\rho}_{r}=0 \\
& \dot{\rho}_{f}^{d}=\frac{1}{l_{d}}\left[\Phi\left(\rho_{f}^{d}\right)-\varphi_{\text {out }}\right] \\
& \dot{l}_{u}=\left\{\begin{array}{lll}
f\left(\rho_{f}^{u}, \rho_{c}\right) & \text { if } l_{u}>l_{r} \\
f\left(\rho_{f}^{u}, \rho_{r}\right)=-v & \text { if } \quad l_{u}=l_{r}
\end{array}\right. \\
& \dot{l}_{r}=\left\{\begin{array}{lll}
f\left(\rho_{c}, \rho_{r}\right)=w & \text { if } \quad l_{u}>l_{r} \\
f\left(\rho_{f}^{u}, \rho_{r}\right)=-v & \text { if } \quad l_{u}=l_{r}
\end{array}\right. \\
& \dot{l}_{d}=f\left(\rho_{r}, \rho_{f}^{d}\right)=-v \text { if } \quad l_{r} \geq l_{d}
\end{aligned}
$$

with initial conditions $\rho_{f}^{u}(0), \rho_{c}(0), \rho_{r}(0)=\rho^{*}, \rho_{f}^{d}(0)$, and $l_{f}^{u}(0)>l_{r}(0)=l_{f}^{d}(0)$.

Remark 5 Because of the particular initial density distribution, and of equations (57)-(58), both densities $\rho_{c}(t)=\rho_{c}(0)$, and $\rho_{r}(t)=\rho^{*}$ remain constant. The rarefaction wave reaches in finite time the upstream free state (i.e. $l_{u}=l_{r}$ ), giving raise to a new shock wave whose amplitude is below the critical density and which moves to the right with velocity $v$, i.e. $i_{u}=i_{r}=-v$. 


\section{DisCUSSION ON THE VLM VERSUS THE LWR AND CTM MODELS}

In this section, we compare the proposed model, namely the VLM, with the LWR model and the CTM, in order to put into evidence similarities and differences. To this end, the dynamic properties of the three models are first analyzed under specific conditions (fully free-flow and fully congested). Then, the capability of the different models to describe the evolution of shockwaves is discussed.

\section{A. Free-flow and congested behavior: I/O-flow maps}

Input-to-Output (I/O) flow maps are suitable tools to compare the different models. These operators describe, in some particular cases, how the boundaries inflows and outflows are related. However, those maps are only defined under the specific conditions of fully free-flow $\left(G_{F}(\mathbf{s}): \varphi_{\text {in }} \mapsto \varphi_{\text {out }}\right)$, or fully congested $\left(G_{C}(\mathbf{s}): \varphi_{\text {out }} \mapsto \varphi_{\text {in }}\right)$, because of the particular downstream/upstream observability property inherent to the fully free/congested modes. In fact, in such modes, the "output" variables ( $\varphi_{\text {out }}$ in the first case, and $\varphi_{i n}$ in the second) are linearly related to the system states.

Lemma 1 (Fully free-flow case) Let the I/O flow map be defined as a linear operator, $\varphi_{\text {out }}(\mathbf{s})=$ $G_{F}(\boldsymbol{s}) \varphi_{\text {in }}(\boldsymbol{s})$, then, $G_{F}(\boldsymbol{s})$ is given as:

i) $G_{F}(\boldsymbol{s})=e^{-\tau_{F} s}$, for the LWR-model

ii) $G_{F}(s)=\frac{1}{\left(\frac{\tau_{F}}{n} s+1\right)^{n}}$, for the CTM

iii) $G_{F}(s) \approx \frac{1}{\left(\tau_{F} s+1\right)}$, for the VLM,

where $n$ is the number of cells of the CTM, $\tau_{F}=L / v$ is the transmission delay in $\left.\mathrm{i}\right)$, while it is the time constant in ii)-iii), and the approximation in the VLM model comes from $L / v \approx(L-\epsilon) / v$.

\section{Proof:}

Condition $i$. In free-flow (2) writes as $\partial_{t} \rho+v \cdot \partial_{x} \rho=0$, and has the solution $\rho(x, t)=\rho^{0}(x-v t)$. The boundary flows can be written as a function of the boundary densities, and hence only as a function of the initial density distribution:

$$
\begin{array}{r}
\varphi_{\text {in }}(t)=v \rho(0, t)=v \rho^{0}(-v t) \\
\varphi_{\text {out }}(t)=v \rho(L, t)=v \rho^{0}(L-v t)
\end{array}
$$


Shifting the outflow of an amount of $L / v$ in time, gives $\varphi_{\text {out }}(t+L / v)=v \rho^{0}(-v t)$. Therefore we have that,

$$
\frac{\varphi_{\text {in }}(t)}{\varphi_{\text {out }}(t+L / v)}=1
$$

or equivalently that, $\varphi_{\text {out }}(t)=\varphi_{\text {in }}(t-L / v)=\varphi_{\text {in }}\left(t-\tau_{F}\right)$, whose Laplace transform is

$$
\varphi_{\text {in }}(\mathbf{s})=e^{-\tau_{F} \mathbf{s}} \varphi_{\text {out }}(\mathbf{s})
$$

Condition $i$. Evaluation of the CTM model (3), with $\Delta l_{i}=L / n$, for $i=1, . . n$, at free-flow gives

$$
\begin{aligned}
\dot{\rho}_{1}(t) & =\frac{v}{\tau_{F} / n}\left(-\rho_{1}(t)+\varphi_{\text {in }}(t)\right) \\
& \vdots \\
\dot{\rho}_{i}(t) & =\frac{v}{\tau_{F} / n}\left(-\rho_{i}(t)+\rho_{i-1}(t)\right) \\
& \vdots \\
\dot{\rho}_{n}(t) & =\frac{v}{\tau_{F} / n}\left(-\rho_{n}(t)+\rho_{n-1}(t)\right) \\
\varphi_{\text {out }}(t) & =v \rho_{n}(t)
\end{aligned}
$$

which describes a forward cascade of $n$ linear first-order filters with transfer function $G_{F}(\mathbf{s})=$ $\frac{1}{\left(\frac{\tau F}{n} \mathbf{s}+1\right)^{n}}$.

Condition iii. The VLM in fully free-flow is given by Equation (38), that is

$$
\begin{aligned}
\dot{\rho}_{f} & =\frac{1}{L-\varepsilon}\left(\varphi_{\text {in }}-v \rho_{f}\right) \approx \frac{1}{\tau_{F}}\left(-\rho_{f}+\frac{\varphi_{\text {in }}}{v}\right) \\
\varphi_{\text {out }}(t) & =v \rho_{f}(t)
\end{aligned}
$$

which describes a linear first-order filter with transfer function $G_{F}(\mathbf{s}) \approx \frac{1}{\left(\tau_{F} \mathbf{s}+1\right)}$.

The dual lemma for the congested case can be stated and proven by analogy with Lemma 1 . In this case, the relevant map of interest is the one from outflows to inflows, as the congestion waves moves upwards (upstream).

Lemma 2 (Fully congested case) Let the O/I flow map be defined as a linear operator, $\varphi_{\text {in }}(\boldsymbol{s})=$ $G_{C}(s) \varphi_{\text {out }}(\boldsymbol{s})+\delta(\boldsymbol{s})$, then, $G_{C}(\boldsymbol{s})$ is given as:

i) $G_{C}(\boldsymbol{s})=e^{-\tau_{C} s}$, and $\delta(s)=0$ for the LWR-model

ii) $G_{C}(\boldsymbol{s})=\frac{1}{\left(\frac{\tau_{C}}{n} \boldsymbol{s}+1\right)^{n}}$, and $\delta(\boldsymbol{s})=w \rho_{M}\left(1-G_{C}\right)$ for the CTM 
iii) $G_{C}(\boldsymbol{s}) \approx \frac{1}{\left(\tau_{C} \boldsymbol{s}+1\right)}$, and $\delta(\boldsymbol{s})=w \rho_{M}\left(1-G_{C}\right)$ for the VLM,

where $\tau_{C}=L / w$ is the transmission time-delay, and the approximation in the VLM model comes from $L / w \approx(L-\epsilon) / w$.

In these special cases, while the LWR behaves as a simple delay line, the CTM approaches the LWR model behavior as the number of cell increases, i.e.

$$
\lim _{n \rightarrow \infty} \frac{1}{\left(\frac{\tau_{F}}{n} \mathbf{s}+1\right)^{n}}=e^{-\tau_{F} \mathbf{s}}
$$

On the other hand, the VLM, in the two considered cases, behaves like a 1-cell CTM. The precision increase which can be obtained with the CTM implies a cost in terms of complexity, in particular in case of large traffic networks. The above analysis confirms the natural intuition one may have about how the models behave in such particular cases. However, those specific cases exclude the shock wave evolution and congestion dynamics. In the next section, we provide some further comparison in several scenarios where congestion and shock occurs.

\section{B. Shock wave behavior}

Traffic jam shock behavior can be studied by considering initial distribution of the form

$$
\rho(l, 0)=\left\{\begin{array}{lll}
\rho^{-} & \text {if } & l<l_{0} \\
\rho^{+} & \text {if } & l>l_{0}
\end{array}\right.
$$

with $l_{0} \in(0, L)$, and with the constant left and right densities, $\rho^{-}, \rho^{+}$, satisfying $0<\rho^{-}<\rho^{*}<$ $\rho^{+}<\rho_{M}$. In this case the shock speed, $\dot{s}$ (in the $l$-direction ${ }^{2}$ ),

$$
\dot{s}=\frac{\Phi\left(\rho^{-}\right)-\Phi\left(\rho^{+}\right)}{\rho^{+}-\rho^{-}}
$$

can be either positive or negative depending on the values of $\rho^{-}, \rho^{+}$. For instance, if $\Phi\left(\rho^{-}\right)-$ $\Phi\left(\rho^{+}\right)>0$, the shock wave propagates upstream increasing the congestion. Conversely, if $\Phi\left(\rho^{-}\right)-\Phi\left(\rho^{+}\right)<0$, the shock wave propagates downstream, and congestion is reduced. With the initial distribution (66), the shock speed is constant, and can be computed analytically from the above equation. The shock propagation in the space-time coordinates $(l, t)$ is then given by

$$
t=\left(l-l_{0}\right) / \dot{s}=\frac{\rho^{+}-\rho^{-}}{\Phi\left(\rho^{-}\right)-\Phi\left(\rho^{+}\right)}\left(l-l_{0}\right)
$$

As illustrated in Fig.6.

\footnotetext{
${ }^{2}$ Note that shock speed in the $x$ natural coordinates writes as $\dot{s}_{x}=\frac{\Phi\left(\rho^{-}\right)-\Phi\left(\rho^{+}\right)}{\rho^{-}-\rho^{+}}$. In the $l$-coordinates the sign of $\dot{s}$ is reversed.
} 


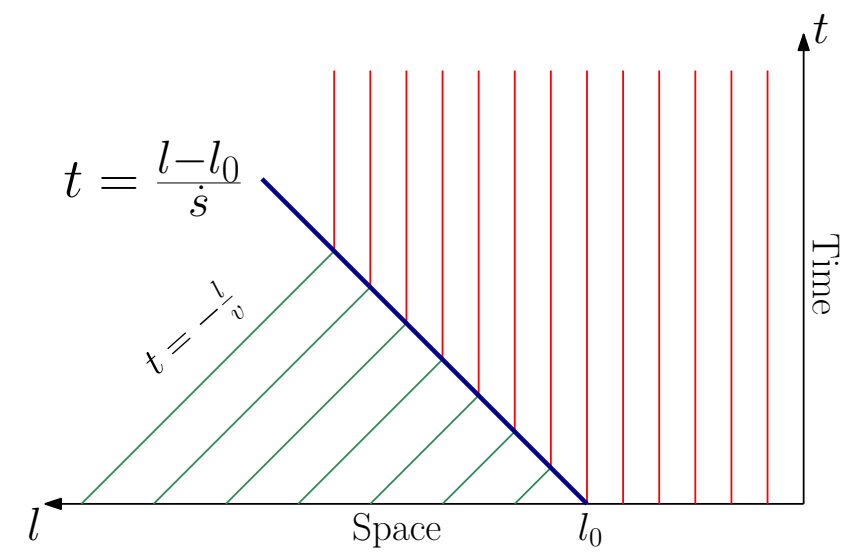

Fig. 6. Illustration of a space-time shock propagation given by the shock line $t=\left(l-l_{0}\right) / \dot{s}$. Congestion starts at location $l=l_{0}$, and propagates backwards. The other lines indicate the motion of the vehicles (particles) with slope $-1 / v$.

The purpose of the following simulation analysis is to evaluate the capability of the VLM model to track the evolution of the shock waves, as well as to compare its behavior with that of the CTM. In the simulations were done under the following conditions:

- LWR (or True solution). Is computed analytically when possible.

- CTM is numerically implemented in matlab using fixed time-steep, by using the standard recursive equations in time. Spatial steeps are of the order of $500 \mathrm{~m}$ each.

- High-order CTM. Is numerically implemented as a CTM model but discretized with a very small spatial steps of the order of $5 \mathrm{~m}$. This leads to a large number of states and is intended for numerical comparisons when the true solutions can not be computed analytically.

- VLM is implemented using standard variable-step solvers in matlab (Simulink).

Simulation parameters are: $L=5 \mathrm{~km}, v=80 \mathrm{Km} / \mathrm{h}, w=20 \mathrm{Km} / \mathrm{h}, \rho^{*}=50 \mathrm{Veh} / \mathrm{Km}$, $\rho_{M}=250 \mathrm{Veh} / \mathrm{Km}, \varphi_{M}=4000 \mathrm{Veh} / \mathrm{h}$. In the left plots of Fig. 7, we illustrate first the consistency between the shock evolution of the original LWR-PDE, named the "true solution", which partitioned the space-time area into the free-flow area in green, and the congested one in red. The plots in the left column of Fig. 7 confirm that the original true solution perfectly coincide with that of the high resolution CTM for both shock traveling directions. The figures at the right confirm that the VLM perfectly tracks the shock waves in this particular case, while the CTM does it in the average with a small phase shift. 

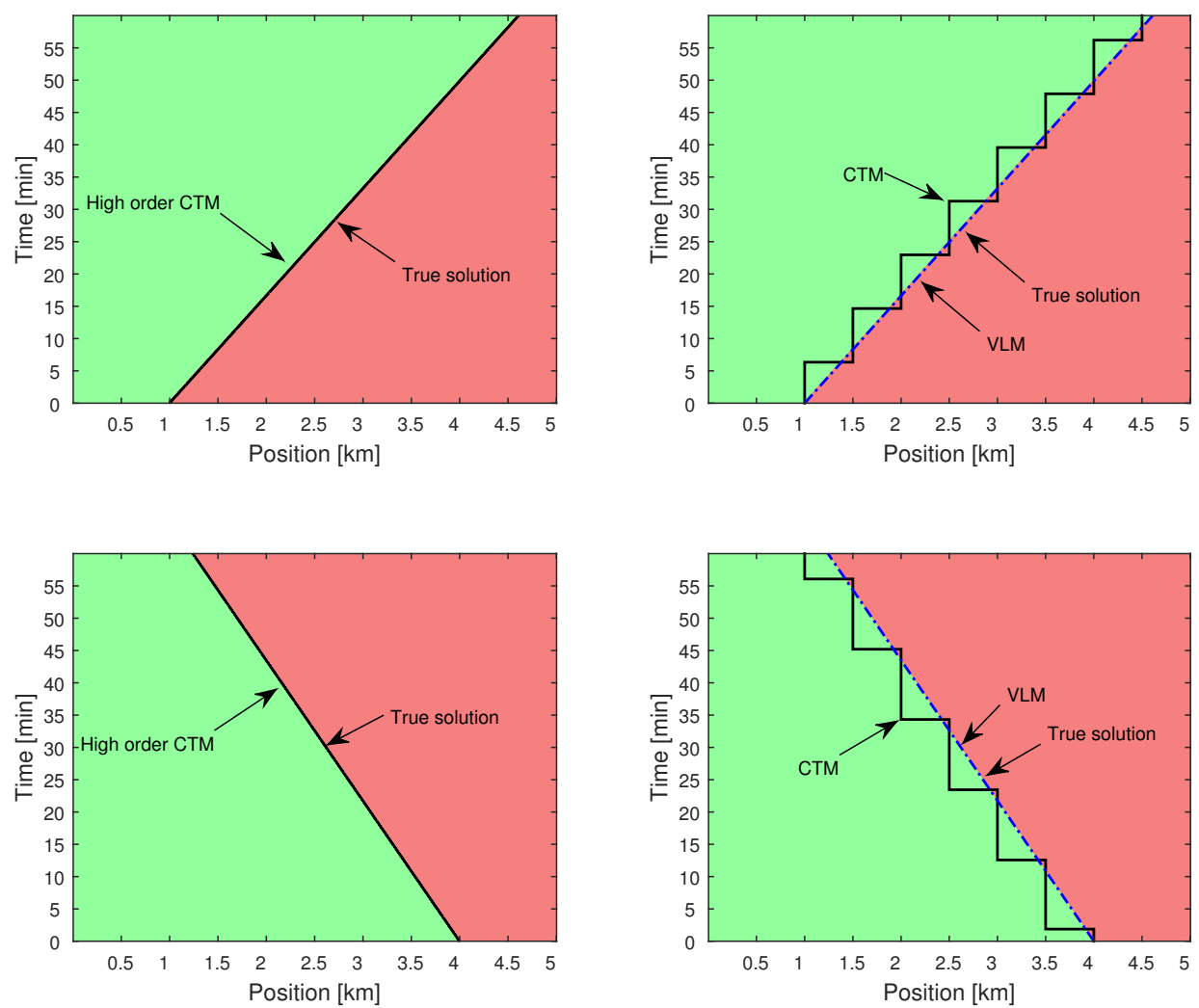

Fig. 7. Simulation of a space-time shock propagation. Left figures show the consistency between the true solution and the one predicted by the high resolution CTM. Right figures compare the true solutions with those of the CTM and the VLM. The upper curves show congestion reduction, with parameters: $\rho^{-}=0.15 \rho^{*}, \rho^{+}=0,75 \rho_{M}, l_{0}=4 L / 5$, and $\dot{s}=-3.611 K m / h$. The lower curves show the congestion spill-over case with parameters: $\rho^{-}=0.5 \rho^{*}, \rho^{+}=0,68 \rho_{M}, l_{0}=L / 5$, and $\dot{s}=2.758 \mathrm{Km} / \mathrm{h}$

\section{Bode-plots for the congestion propagation}

As shown in the previous simulations, the VLM and to some extent the CTM well describe the shock wave propagation. However, both models are inherently low-order and some low-pas filtering effects are to be expected. The purpose of this subsection is to evaluate such attenuation and understand the cut-off frequency spectrum of the models. The simulations are conducted starting from the initial conditions at the equilibrium: $\dot{\rho}_{f}=\dot{\rho}_{c}=\dot{l}=0$, and with $l(0)=L / 2$. In addition, the initial condition for the congested part is selected so that the congested density remains time-invariant, i.e. $\rho_{c}(0)=\rho_{M}-\frac{\varphi_{M}}{2 w}>0 \Longrightarrow \rho_{c}(t)=\rho_{c}(0)$. The outflow was limited by a bottleneck at $\varphi_{\text {out }}=\varphi_{M} / 2$, while the inflow was periodic $\varphi_{\text {in }}=\varphi_{M} / 2+A(\omega) \sin (\omega t)$. 

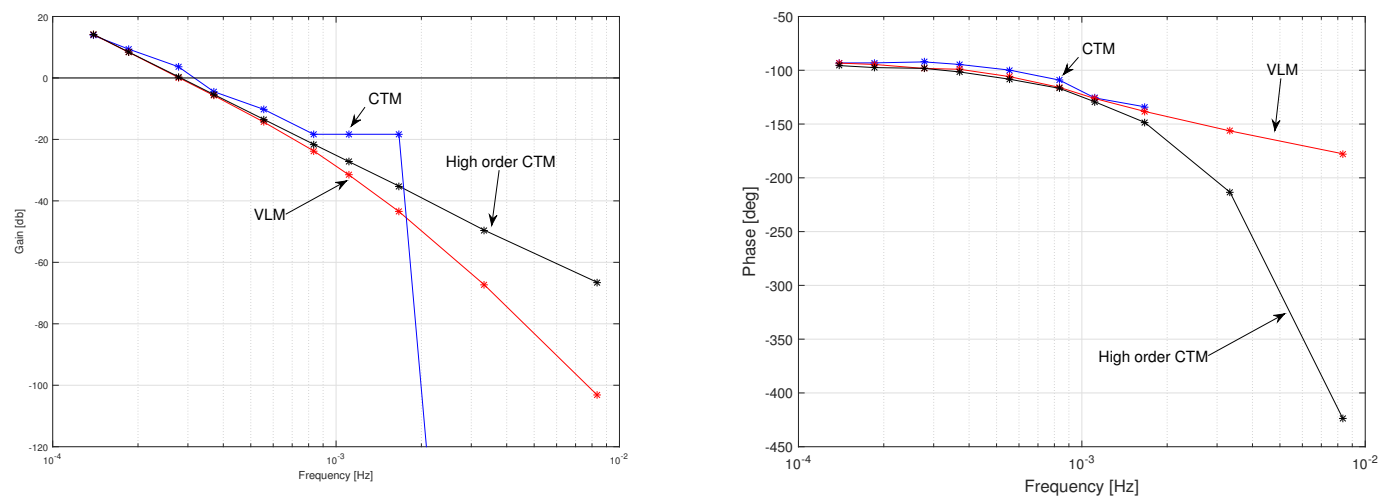

Fig. 8. Bode plots for the map $\varphi_{i n} \mapsto l$ for the VLM, and maps between $\varphi_{i n}$ and the front congestion wave for the CTM and the high resolution CTM.

$A(\omega)$ was selected, so that the variation of $l$ remains within the natural boundaries $(0, L)$ to preserve a certain degree of linearity of the analysis. Note also that under such conditions, the map $\varphi_{i n} \mapsto l$ can be approximated by

$$
l \approx \frac{K}{\mathbf{s}(\tau \mathbf{s}+1)} \varphi_{i n}
$$

with $K \approx 1 /\left(\bar{\rho}_{c}-\bar{\rho}_{f}\right)$, and $\tau=(L-\bar{l}) / v$, where $\left(\bar{\rho}_{c}, \bar{\rho}_{f}, \bar{l}\right)$ are the values around which the linearization is performed.

Fig. 8 shows the Bode plots of the map between the inflow and the congestion front. These plots depict the frequency response of the map, i.e. the magnitude and phase variation of the complex-valued function which describes the considered map as a function of the frequency. Several observations are in order. First, it is worth noting that magnitude and phase plots of both the CTM and the VLM well match the Bode locus of the true solution (represented here by the high resolution CTM) at low frequencies. Then, around $10^{-3} \mathrm{~Hz}$, the CTM saturates in gain and phase, and finally its gain dramatically drops to zero just thereafter. This means that the CTM fails to suitably track the shown waves at medium-high frequencies. On the other hand, the VLM does not display such behavior, but it shows a discrepancy with respect to the true solution. As expected, the discrepancy increases as the frequency grows because of the low-pass filtering effect of the VLM. 


\section{CAse Studies}

The practical utility of the proposed model is now illustrated by reference to two case studies: a ring road and a urban road with traffic lights. they are discussed in the next two subsections, respectively, by making reference to simulation results.

\section{A. Case study 1: Ring-road scenario}
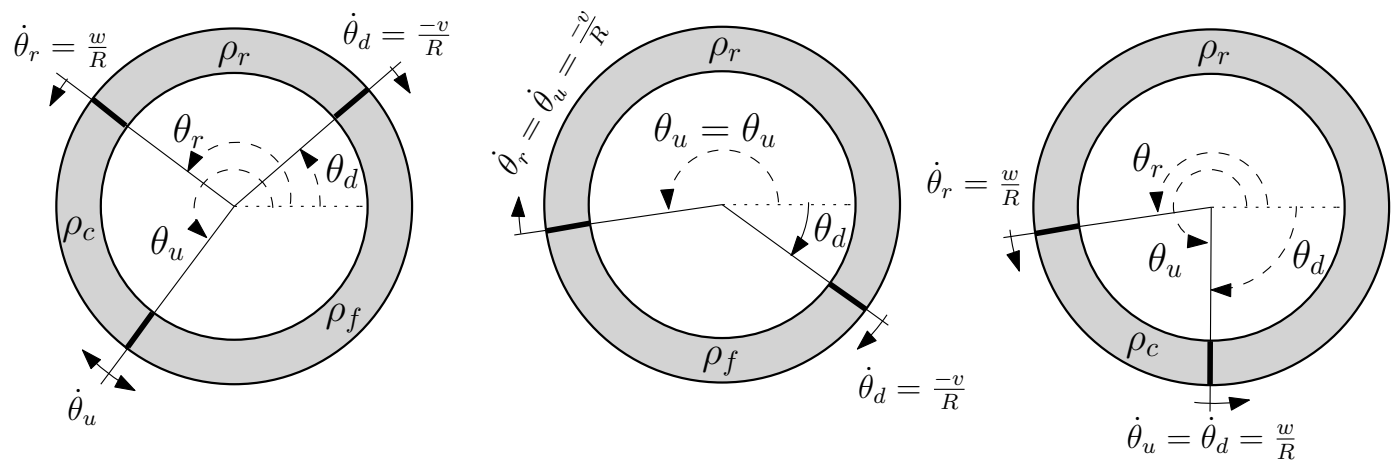

Fig. 9. Illustration of the ring-road scenario. Left figure shown the scenario with the associated variables. Center and right figures show the two possible equilibria

In this subsection, a case study of a closed ring-road scenario shown in Fig. 9 is presented. We first give the main model control properties, and then we show how those properties can be used for variable speed optimization.

Specifically, the ring-road in Fig. 9 is considered, with $l_{u}=R \theta_{u}, l_{r}=R \theta_{r}, l_{d}=R \theta_{d}$, $\rho_{f}^{u}=\rho_{f}^{d}=\rho_{f}, \varphi_{\text {in }}=\varphi_{\text {out }}$. Model (56)-(62), in the set $\Omega_{\theta}=\left\{\left(\theta_{u}, \theta_{r}, \theta_{d}\right): \theta_{u} \geq \theta_{r} \geq \theta_{d}\right\}-$ 
$\left\{\left(\theta_{u}, \theta_{r}, \theta_{d}\right): \theta_{u}=\theta_{r}=\theta_{d}\right\}$, can be written in the circular coordinates as,

$$
\begin{aligned}
\dot{\rho}_{f} & =0, \quad \rho_{f}(0)=\rho_{f}^{0} \\
\dot{\rho}_{c} & =0, \quad \rho_{c}(0)=\rho_{c}^{0} \\
\dot{\rho}_{r} & =0, \quad \rho_{r}(0)=\rho_{f}^{*} \\
\dot{\theta}_{u}=\frac{l_{u}}{R} & =\frac{1}{R} \begin{cases}f\left(\rho_{f}, \rho_{c}\right) & \text { if } \theta_{u}>\theta_{r} \\
f\left(\rho_{f}, \rho_{r}\right)=-v & \text { if } \theta_{u}=\theta_{r}\end{cases} \\
\dot{\theta}_{r}=\frac{\dot{l}_{r}}{R} & =\frac{1}{R} \begin{cases}f\left(\rho_{c}, \rho_{r}\right)=w & \text { if } \theta_{u}>\theta_{r} \\
f\left(\rho_{f}, \rho_{r}\right)=-v & \text { if } \theta_{u}=\theta_{r} \\
f\left(\rho_{r}, \rho_{f}\right)=-v & \text { if } \theta_{r} \geq \theta_{d} \\
f\left(\rho_{r}, \rho_{c}\right)=w & \text { if } \theta_{d}=\theta_{u}\end{cases} \\
\dot{\theta}_{d}=\frac{\dot{l}_{d}}{R} & =\frac{1}{R} \begin{cases}R\end{cases}
\end{aligned}
$$

with initial conditions $\left(\theta_{u}^{0}, \theta_{r}^{0}, \theta_{d}^{0}\right) \in \Omega_{\theta}$.

1) Equilibrium points, stability and optimal speed limits design: The model (67)-(72), has in general two classes of equilibrium points depending on the initial conditions. One class (Class A) is composed of one part in free-flow and the other at the critical density, while the second class (Class B) consists of one part in congested mode, and the other at the critical density, see Fig. 9. Consider the following definitions:

$$
\begin{aligned}
\Delta \theta_{f}(t) & =2 \pi+\theta_{d}(t)-\theta_{u}(t) \\
\Delta \theta_{c}(t) & =\theta_{u}(t)-\theta_{r}(t) \\
\Delta \theta_{*}(t) & =\theta_{r}(t)-\theta_{d}(t)
\end{aligned}
$$

with the notation $\Delta \theta_{(\cdot)}(0)=\Delta \theta_{(\cdot)}^{0}$.

Lemma 3 Consider the ring-road model (67)-(72), with initial conditions $\theta_{u}^{0}>\theta_{r}^{0} \geq \theta_{d}^{0}$. Let

$$
\begin{gathered}
a=\frac{\rho_{f}^{0}}{\rho_{c}^{0}-\rho_{f}^{0}}, \quad b=\frac{\Phi\left(\rho_{c}^{0}\right)}{\rho_{c}^{0}-\rho_{f}^{0}}, \quad \beta=\frac{\Delta \theta_{f}^{0}}{\Delta \theta_{c}^{0}} \\
c(\beta, a, b)=\frac{b(1+\beta)+\beta w}{a(1+\beta)+1}, \quad f_{0}=f\left(\rho_{f}^{0}, \rho_{c}^{0}\right)
\end{gathered}
$$

Then:

A) The system converges in a finite time $t_{1}=R \frac{\Delta \theta_{c}^{0}}{w-f_{0}}$, to the Class-A equilibrium points if initial conditions satisfy:

$$
\frac{f_{0}+v}{w-f_{0}}<\beta
$$


with the final angles given as:

$$
\begin{aligned}
& \Delta \theta_{c}\left(t_{1}\right)=0 \\
& \Delta \theta_{f}\left(t_{1}\right)=\Delta \theta_{f}^{0}-\frac{v+f_{0}}{w-f_{0}} \Delta \theta_{c}^{0} \\
& \Delta \theta_{*}\left(t_{1}\right)=\Delta \theta_{*}^{0}+\frac{v+w}{w-f_{0}} \Delta \theta_{c}^{0}
\end{aligned}
$$

with $\Delta \theta_{f}\left(t_{1}\right)+\Delta \theta_{*}\left(t_{1}\right)=2 \pi$.

$B)$ The system converges in a finite time $t_{2}=R \frac{\Delta \theta_{f}^{0}}{v+f_{0}}$, to the Class-B equilibrium points if initial conditions satisfy:

$$
\frac{f_{0}+v}{w-f_{0}}>\beta
$$

with the final angles given as:

$$
\begin{aligned}
& \Delta \theta_{c}\left(t_{2}\right)=\Delta \theta_{c}^{0}-\frac{w-f_{0}}{v+f_{0}} \Delta \theta_{f}^{0} \\
& \Delta \theta_{f}\left(t_{2}\right)=0 \\
& \Delta \theta_{*}\left(t_{2}\right)=\Delta \theta_{*}^{0}+\frac{v+w}{v+f_{0}} \Delta \theta_{f}^{0}
\end{aligned}
$$

with $\Delta \theta_{c}\left(t_{2}\right)+\Delta \theta_{*}\left(t_{2}\right)=2 \pi$.

Proof. The proof is straightforward by computing the angle evolutions of the solutions

$$
\begin{aligned}
& \theta_{r}(t)=\theta_{r}^{0}+\frac{w}{R} t \\
& \theta_{u}(t)=\theta_{u}^{0}+\frac{f_{0}}{R} t \\
& \theta_{d}(t)=\theta_{d}^{0}-\frac{v}{R} t
\end{aligned}
$$

and noticing that $f_{0}$ can be rewritten as

$$
f_{0}=\frac{\phi\left(\rho_{f}^{0}\right)-\Phi\left(\rho_{c}^{0}\right)}{\rho_{c}^{0}-\rho_{f}^{0}}=v \frac{\rho_{f}^{0}}{\rho_{c}^{0}-\rho_{f}^{0}}-\frac{\Phi\left(\rho_{c}^{0}\right)}{\rho_{c}^{0}-\rho_{f}^{0}}=a v-b
$$

For Class $A$, we require the existence of a finite time $t_{1}$ such that: $\left.i\right) \theta_{r}\left(t_{1}\right)=\theta_{u}\left(t_{1}\right)$, and ii) $\theta_{u}\left(t_{1}\right)<2 \pi+\theta_{d}\left(t_{1}\right)$. Now, from the above solutions, we get

$$
\begin{aligned}
& \theta_{r}^{0}+\frac{w}{R} t_{1}=\theta_{u}^{0}+\frac{f_{0}}{R} t_{1} \\
& \theta_{u}^{0}+\frac{f_{0}}{R} t_{1}<2 \pi+\theta_{d}^{0}-\frac{v}{R} t_{1}
\end{aligned}
$$

Then, $t_{1}$ is obtained from the first equation. The velocity range comes from the second equations where we replace $t_{1}$, and noting the fact that $f_{0}<0$, and hence $a v-b<0$. 


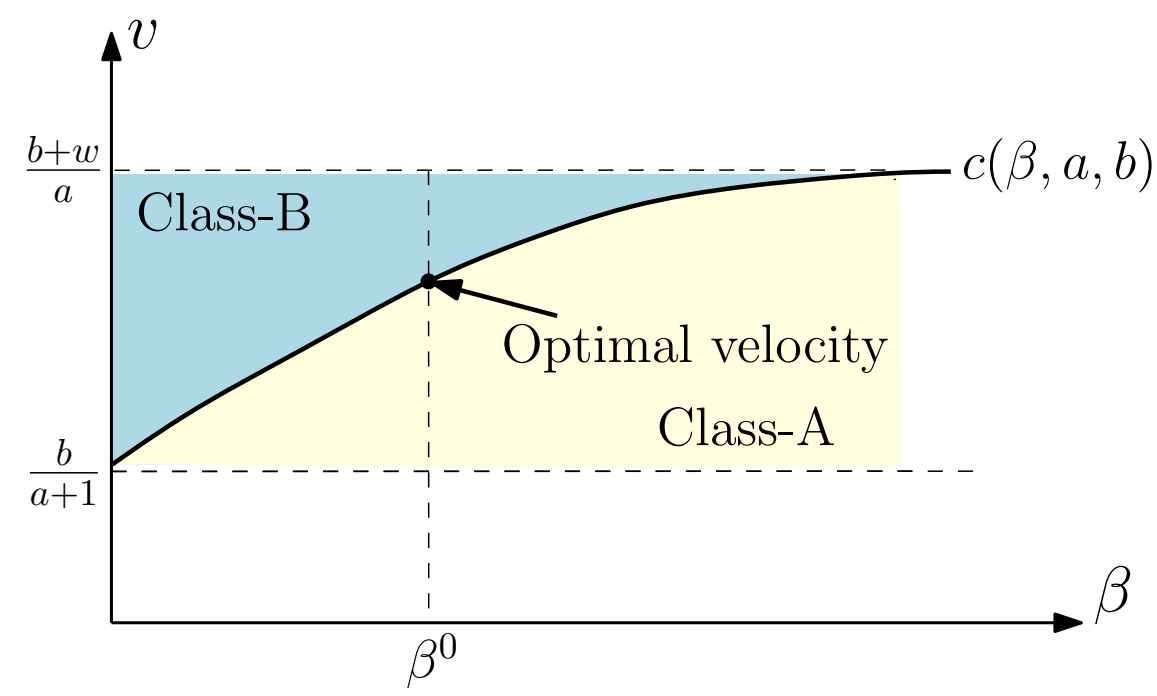

Fig. 10. Admissible velocity domain as a function of the initial conditions. The domain is limited by $v \in\left(v_{m}, v_{M}\right)$, where $v_{m}=b /(a+1), v_{M}=(b+w) / a$

For Class $B$, we require the existence of a finite time $t_{2}$ such that: $\left.i\right) \theta_{u}\left(t_{2}\right)=2 \pi+\theta_{d}\left(t_{2}\right)$, and $i$ ) $\theta_{u}\left(t_{2}\right)>\theta_{r}\left(t_{2}\right)$. Now, from the above solutions, we get

$$
\begin{aligned}
& \theta_{u}^{0}+\frac{f_{0}}{R} t_{2}=2 \pi+\theta_{d}^{0}-\frac{v}{R} t_{2} \\
& \theta_{u}^{0}+\frac{f_{0}}{R} t_{2}>\theta_{r}^{0}+\frac{w}{R} t_{2}
\end{aligned}
$$

As before, $t_{2}$ is obtained from the first equation. The velocity range comes from the second equation where we replace $t_{2}$.

Fig. 10 shows the velocity admissible domain as a function of the initial angles defined by $\beta$. Note this domain corresponds to free-flow velocities which are consistent with the model densities definition. For instance, the lower admissible value $v_{m}=b /(a+1)$ is equal to $\Phi\left(\rho_{c}^{0}\right) / \rho_{c}^{0}$, and maximum velocity bound $v_{M}=(b+w) / a$ is equal to $-w\left(1-\rho_{M} / \rho_{f}^{0}\right)$. As long as $v \in$ $\Omega_{v}=\left(v_{m}, v_{M}\right)$ the evolution of $\rho_{f}(t), \rho_{c}(t)$ will remain in the corresponding free and congested part of the fundamental diagram. The curve $c(\beta, a, b)$ is the velocity boundary between the two possible equilibria classes.

Note that this allows for some flexibility in the selection of $v \in \Omega_{v}$, and therefore opens the possibility to optimize the network operation. For instance by minimizing the lap time, i.e.

$$
v_{o p t}=\min _{v \in \Omega_{v}, i \in 1,2} J(v, i)
$$


where

$$
J(v, i)=R\left[\frac{\Delta \theta_{*}\left(t_{i}\right)}{v}+\frac{\Delta \theta_{f}\left(t_{i}\right)}{v}+\frac{\Delta \theta_{c}\left(t_{i}\right)}{v_{m}}\right] .
$$

A particular case of interest is to find the optimal free-speed for the Class-A equilibria, which can be easily computed by observing Fig. 10, that is

$$
v_{o p t}=\min _{v \in \Omega_{v}, i=1} J(v, 1)=c(\beta, a, b)
$$

leading to $J\left(v_{\text {opt }}, 1\right)=2 \pi R / c(\beta, a, b)$.

TABLE I

SIMULATION PARAMETERS

\begin{tabular}{|c|c|c|c|}
\hline Parameter & Description & Value & Unit \\
\hline \hline$L$ & section length & 1 & $\mathrm{~km}$ \\
\hline$v$ & free-flow speed & 80 & $\mathrm{~km} / \mathrm{h}$ \\
\hline$w$ & congestion propagation speed & 20 & $\mathrm{~km} / \mathrm{h}$ \\
\hline$\rho_{M}$ & jam density & 250 & $\mathrm{veh} / \mathrm{km}$ \\
\hline$\rho^{*}$ & critical density & 50 & $\mathrm{veh} / \mathrm{km}$ \\
\hline$\varphi_{M}$ & maximum flow & 4000 & $\mathrm{veh} / \mathrm{h}$ \\
\hline$\rho_{f}^{0}$ & initial free-flow cell density & 30 & $\mathrm{veh} / \mathrm{km}$ \\
\hline$\rho_{c}^{0}$ & initial congested cell density & 150 & $\mathrm{veh} / \mathrm{km}$ \\
\hline$l_{0}$ & initial congestion length & {$[0.4 L, 0.4 L, 0.2 L]$} & $\mathrm{km}$ \\
\hline
\end{tabular}

2) Simulation results: The two possible cases (A and B) mentioned in Subsection VII-A1 can be reproduced in simulation. The considered physical parameters of the model are given in Table VII-A1. The initial conditions are $\rho_{f}^{0}=30 v e h / k m, \rho_{c}^{0}=150 v e h / k m$ for case A, and $\rho_{f}^{0}=10 \mathrm{veh} / \mathrm{km}, \rho_{c}^{0}=100 \mathrm{veh} / \mathrm{km}$ for case B. In both cases the radius is equal to $R=0.8 \mathrm{~km}$, and the initial congestion length is $l_{u}=\frac{2 \pi}{3} R=1.67 \mathrm{~km}$ (with $\theta_{u}(0)=\frac{2 \pi}{3}$, and $\left.\theta_{r}(0)=0\right)$. Vehicles are assumed to flow in a clockwise direction. Due to these initial conditions, in case A the rotation speed is $\dot{\theta}_{u}=3.33 \mathrm{~km} / \mathrm{h}$, while in case B is $\dot{\theta}_{u}=-24.44 \mathrm{~km} / \mathrm{h}$. Results are shown in Fig. 11 for both cases. Six different snapshots are reported. From them, it is possible to observe how the rarefaction process evolves and the reached equilibrium. As expected, two possible equilibria may occur. In case $\mathrm{A}$, after that the rarefaction wave is produced, $\theta_{u}$ reaches $\theta_{r}$ and the equilibrium is given by a zone at $\rho^{*}$ and by one at $\rho_{f}^{0}$. In case $\mathrm{B}, \theta_{u}$ reaches $\theta_{d}$ and the equilibrium is given by a zone at $\rho_{c}^{0}$ and by one at $\rho^{*}$. 


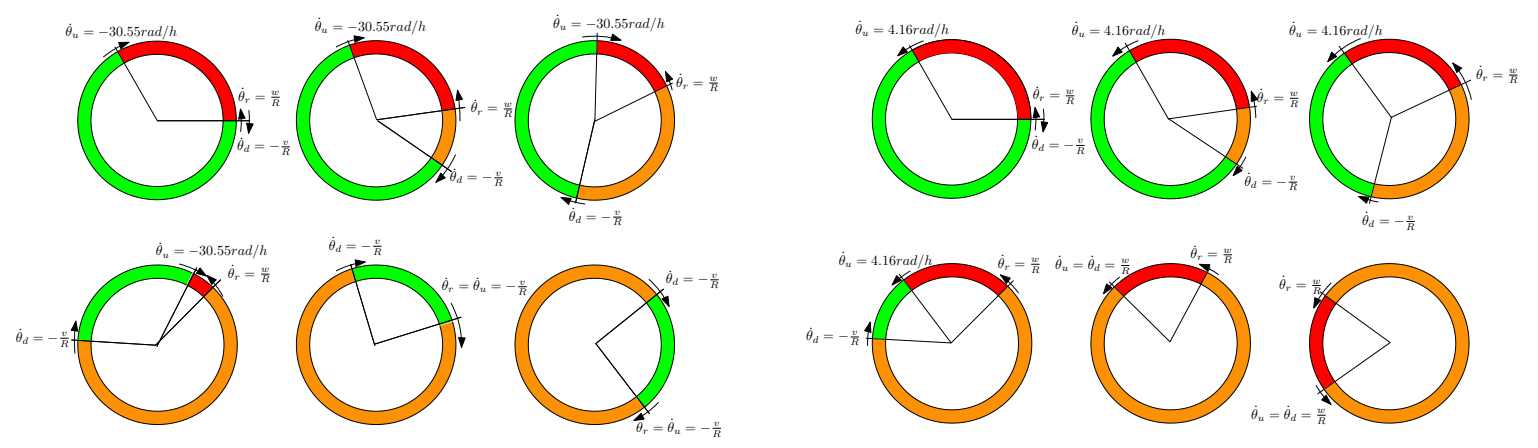

Fig. 11. Snapshots of time evolution of the ring road traffic: left figures show the results in case A, whereas right figures those in case B. Free-flow zones are indicated in green, critical-density zones in orange and congested zones in red.

\section{B. Case Study 2: urban road with traffic lights}

This second scenario corresponds to a classical urban homogeneous road including traffic lights at the upstream and downstream extremes.

Before proceeding to analyze this second case study, it is useful to observe that it is possible to approximate the two-shock waves (51) by a single shock by using the so-called "equal area rule", as shown in Fig. 12. This is formally stated in the theorem below.

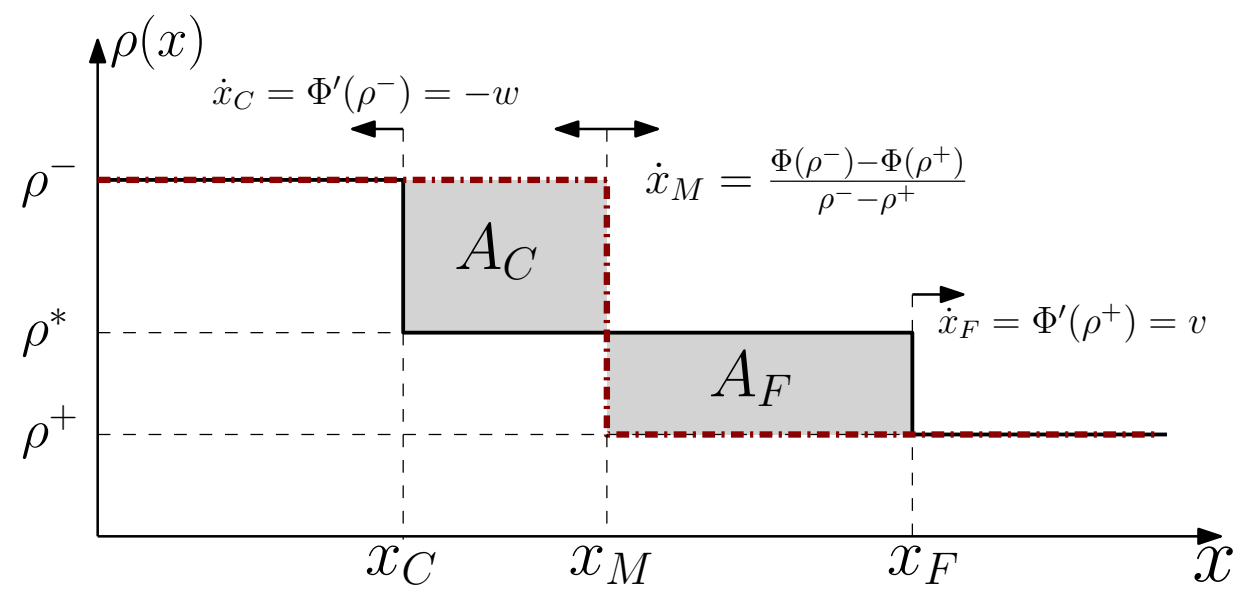

Fig. 12. Illustration of the approximation of the three-valued solution (solid line) of the rarefaction wave by a single shock at $x_{M}$ using the equal area rule (dashed-doted line). 
Theorem 2 Consider a triangular piece-wise fundamental diagram $\Phi(\rho)$, and a distribution of the form shown in Fig. 4, with a unique solution at the downstream boundary $x_{D}$ given by (51). Then the two-shock waves evolution can be approximated by an uniquely defined single shock located at the point $x_{M}$ with velocity

$$
\dot{x}_{M}=\frac{\Phi\left(\rho^{-}\right)-\Phi\left(\rho^{+}\right)}{\rho^{-}-\rho^{+}}
$$

The approximation holds in the sense that the area $A_{C}$ (number of vehicles) at the congested part separated by $x_{M}$ equals the area (number of vehicles) at the free part $A_{F}$.

Proof 1 Define the areas $A_{C}=\Delta \rho_{C} \Delta x_{C}$, and $A_{F}=\Delta \rho_{F} \Delta x_{F}$, with $\Delta \rho_{C}=\rho^{-}-\rho^{*}, \Delta \rho_{F}=$ $\rho^{*}-\rho^{+}, \Delta x_{C}=x_{M}-x_{C}, \Delta x_{F}=x_{F}-x_{M}$. Imposing the "equal area rule" $A_{C}=A_{F}$, with $A_{C}(x, 0)=A_{F}(x, 0)=0$ is equivalent to $\dot{A}_{C}=\dot{A}_{F}$, which yields,

$$
\Delta \rho_{C} \frac{d}{d t} \Delta x_{C}=\Delta \rho_{F} \frac{d}{d t} \Delta x_{F}
$$

noticing that the wave velocities at the edges are: $\frac{d x_{C}}{d t}=\Phi^{\prime}\left(\rho^{-}\right)$, and $\frac{d x_{F}}{d t}=\Phi^{\prime}\left(\rho^{+}\right)$, we have

$$
\frac{d}{d t} \Delta x_{C}=\dot{x}_{M}-\Phi^{\prime}\left(\rho^{-}\right), \quad \text { and } \quad \frac{d}{d t} \Delta x_{F}=\Phi^{\prime}\left(\rho^{+}\right)-\dot{x}_{M}
$$

replacing this expression into the one above, with some simplification, gives,

$$
\dot{x}_{M}=\frac{\Phi^{\prime}\left(\rho^{-}\right)\left(\rho^{-}-\rho^{*}\right)-\Phi^{\prime}\left(\rho^{+}\right) \rho^{+}}{\rho^{-}-\rho^{+}}
$$

using the fact that $\Phi(\rho)$ has a maximum at $\rho=\rho^{*}$ where the two lines intersect, i.e. $\Phi^{\prime}\left(\rho^{+}\right) \rho^{*}=$ $\Phi^{\prime}\left(\rho^{-}\right)\left(\rho^{*}-\rho_{M}\right)$, the above equations simplifies to

$$
\dot{x}_{M}=\frac{\Phi\left(\rho^{-}\right)-\Phi\left(\rho^{+}\right)}{\rho^{-}-\rho^{+}}
$$

where we have used the equalities: $\Phi\left(\rho^{-}\right)=\Phi^{\prime}\left(\rho^{-}\right)\left(\rho^{-}-\rho_{M}\right)$, and $\Phi\left(\rho^{+}\right)=\Phi^{\prime}\left(\rho^{+}\right) \rho^{+}$.

Remark 6 Note that for the triangular form, $\dot{x}_{M}=\frac{-w\left(\rho^{-}-\rho_{M}\right)-v \rho^{+}}{\rho^{-}-\rho^{+}}$is constant. Its direction is determined by the sign of the difference between left and right flows at the initial distribution. Finally note that $\dot{x}_{M}$ satisfies the Rankine-Hugoniot jump condition. The approximation can thus be understood as a shock located at $x_{M}(t)=x_{M}(0)+\dot{x}_{M} t$, with $x_{M}(0)=x_{D}$.

Making reference to Fig. 13, the following variables and definitions are introduced.

- $(\cdot)^{i}$ - index used to denote the considered $i$-section. Sections are regarded in increasing order in the downstream direction. For simplicity in the notation, indexes will only be used to indicate sections other than the current $i$-section, i.e. $l^{i}=l, l^{(i+1)}=l^{(i+1)}$, etc. 


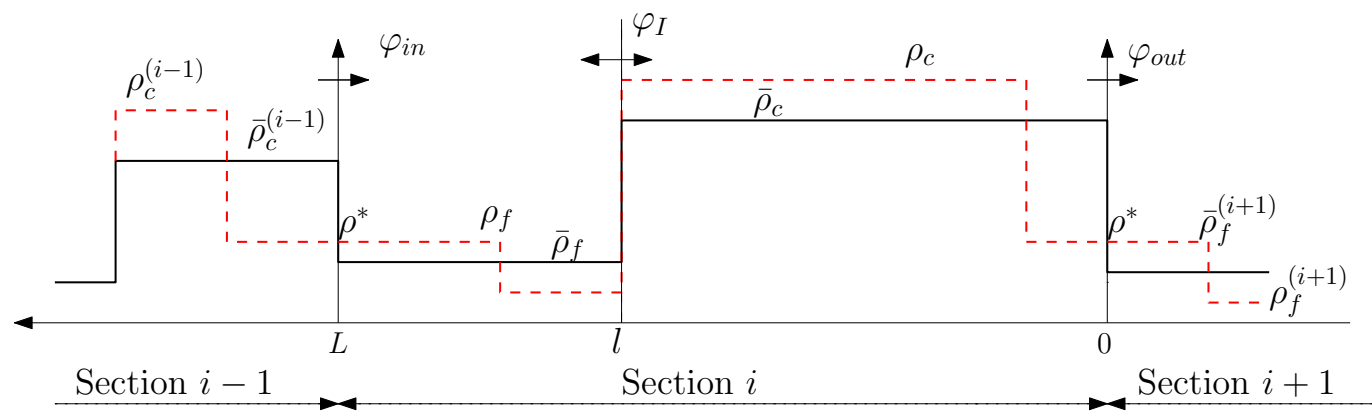

Fig. 13. Illustration of the real solution with upstream/downstream rarefaction waves (solid line), and the proposed approximation of the rarefaction (dashed-red line).

- $\left(\varphi_{\text {in }}, \varphi_{\text {out }}, \varphi_{I}\right)$, are the fixed in/out flows, and the moving interface flow of the $i^{\text {th }}$-section, respectively.

- $\left(\rho_{f}, \rho_{c}\right)$ - are the density distributions of the $i^{\text {th }}$-section as original produced by the rarefaction waves

- $\left(\bar{\rho}_{f}, \bar{\rho}_{c}\right)$ - are the averaged densities of the $i^{\text {th }}$-section, including the effects of the multiple rarefaction waves.

- $\alpha \in\{0,1\}$ is the traffic light boolean variable. $\alpha=1$ indicates green light, whereas $\alpha=0$ indicates red light.

- $t_{k}$ is the time-instant when the rarefaction wave starts at the downstream part of the $i^{t h}$ section. It correspond to the time instant when the green light is turned on, i.e. $\alpha: 0 \rightarrow 1$, or equivalently when $\Delta \alpha_{k}=\alpha_{k}-\alpha_{k-1}=1$.

- $\mathcal{C}_{k}$ is the rarefaction wave triggering condition for the $i^{t h}$-section. $\overline{\mathcal{C}}_{k}$ is its negation. It can be written in several forms. We propose the following one:

$$
\mathcal{C}=\left\{\left(\rho_{c}>\rho^{*}\right) \bigcap\left(\varphi_{\text {out }}=\varphi_{M}\right) \bigcap(\alpha=1)\right\}
$$

with $t_{k}=\left\{t \geq 0\right.$, s.t. $\left.\mathcal{C} \cap\left(\Delta \alpha_{k}=1\right)\right\}, \forall k \geq 1$.

In this case the model can be written relying on the following set of equations

$$
\begin{aligned}
\dot{\bar{\rho}}_{f} & =\frac{1}{L-l}\left[\varphi_{\text {in }}+f\left(\rho^{-}, \rho^{+}\right)\left(\bar{\rho}_{f}-\rho^{-}\right)-\Phi\left(\rho^{-}\right)\right] \\
\dot{\bar{\rho}}_{c} & =\frac{1}{l}\left[-\varphi_{\text {out }}+f\left(\rho^{-}, \rho^{+}\right)\left(\rho^{+}-\bar{\rho}_{c}\right)+\Phi\left(\rho^{+}\right)\right] \\
\dot{l} & =f\left(\rho^{-}, \rho^{+}\right)=\frac{\Phi\left(\rho^{+}\right)-\Phi\left(\rho^{-}\right)}{\rho^{-}-\rho^{+}}
\end{aligned}
$$


with

$$
\begin{aligned}
\varphi_{\text {in }} & =\alpha \cdot \min \left\{D\left(\bar{\rho}_{c}^{(i-1)}\right), S\left(\bar{\rho}_{f}^{i}\right)\right\} \\
\varphi_{\text {out }} & =\alpha \cdot \min \left\{D\left(\bar{\rho}_{c}^{i}\right), S\left(\bar{\rho}_{f}^{(i+1)}\right)\right\}
\end{aligned}
$$

where $\rho^{-}, \rho^{+}$are the left and right density at $l$, given by a

$$
\left(\rho^{-}, \rho^{+}\right)=\left\{\begin{array}{lll}
\left(\bar{\rho}_{f}, \bar{\rho}_{c}\left(t_{k}^{i}\right)\right) & \text { if } & \mathcal{C}^{i} \cap \overline{\mathcal{C}}^{(i-1)} \\
\left(\bar{\rho}_{f}\left(t_{k}^{(i-1)}\right), \bar{\rho}_{c}\right) & \text { if } & \overline{\mathcal{C}}^{i} \cap \mathcal{C}^{(i-1)} \bigcap\left(\bar{\rho}_{f}<\rho^{*}\right) \\
\left(\bar{\rho}_{f}\left(t_{k}^{(i-1)}\right), \bar{\rho}_{c}\left(t_{k}^{i}\right)\right) & \text { if } & \mathcal{C}^{i} \cap \mathcal{C}^{(i-1)} \bigcap\left(\bar{\rho}_{f}<\rho^{*}\right) \\
\left(\bar{\rho}_{f}, \bar{\rho}_{c}\right) & \text { else } &
\end{array}\right.
$$

The different definitions for the boundary densities, and hence for the time-evolution of the congestion fronts, correspond to the different cases included in this scenario. The first 3 cases are the following: i) the rarefaction occurs at the downstream boundary of the the current section, ii) the rarefaction wave is produced at the previous section, and iii) the rarefaction wave takes place at both the current and the previous section. The last case corresponds to the situation when one of the extreme of the rarefaction wave reaches the point $l$. In this case, the section becomes either fully-free or fully-congested. The various cases are illustrated in Fig. 14. The details of the model derivation are given next. The average model (73)-(78) is derived from the vehicle conservation equations, with $\bar{N}_{f}=(L-l) \bar{\rho}_{f}$, and $\bar{N}_{c}=l \bar{\rho}_{c}$, constrained with the following two conditions:

a) Same amount of vehicles. That is $\bar{N}_{f}=N_{f}$, and $\bar{N}_{c}=N_{c}$. This constraint is ensured if the in/out flows of the sections are the same for both the real and the approximated solution.

b) The time-evolution of the shock wave $l$ should be the same for both the real and the approximated solution.

The rate of vehicles variations can now be computed as

$$
\left.\begin{array}{c}
\dot{\bar{N}}_{f}=\varphi_{\text {in }}-\varphi_{I} \\
\dot{\bar{N}}_{c}=\varphi_{I}-\varphi_{\text {out }}
\end{array}\right\} \Rightarrow \begin{aligned}
-i \bar{\rho}_{f}+(L-l) \dot{\bar{\rho}}_{f} & =\varphi_{\text {in }}-\varphi_{I} \\
i \bar{\rho}_{c}+l \dot{\bar{\rho}}_{c} & =\varphi_{I}-\varphi_{\text {out }}
\end{aligned}
$$

and the state-variable representation is given by

$$
\begin{aligned}
& \dot{\bar{\rho}}_{f}=\frac{1}{L-l}\left[\varphi_{\text {in }}+f\left(\rho^{-}, \rho^{+}\right) \bar{\rho}_{f}-\varphi_{I}\right] \\
& \dot{\bar{\rho}}_{c}=\frac{1}{l}\left[-\varphi_{\text {out }}-f\left(\rho^{-}, \rho^{+}\right) \bar{\rho}_{c}+\varphi_{I}\right]
\end{aligned}
$$




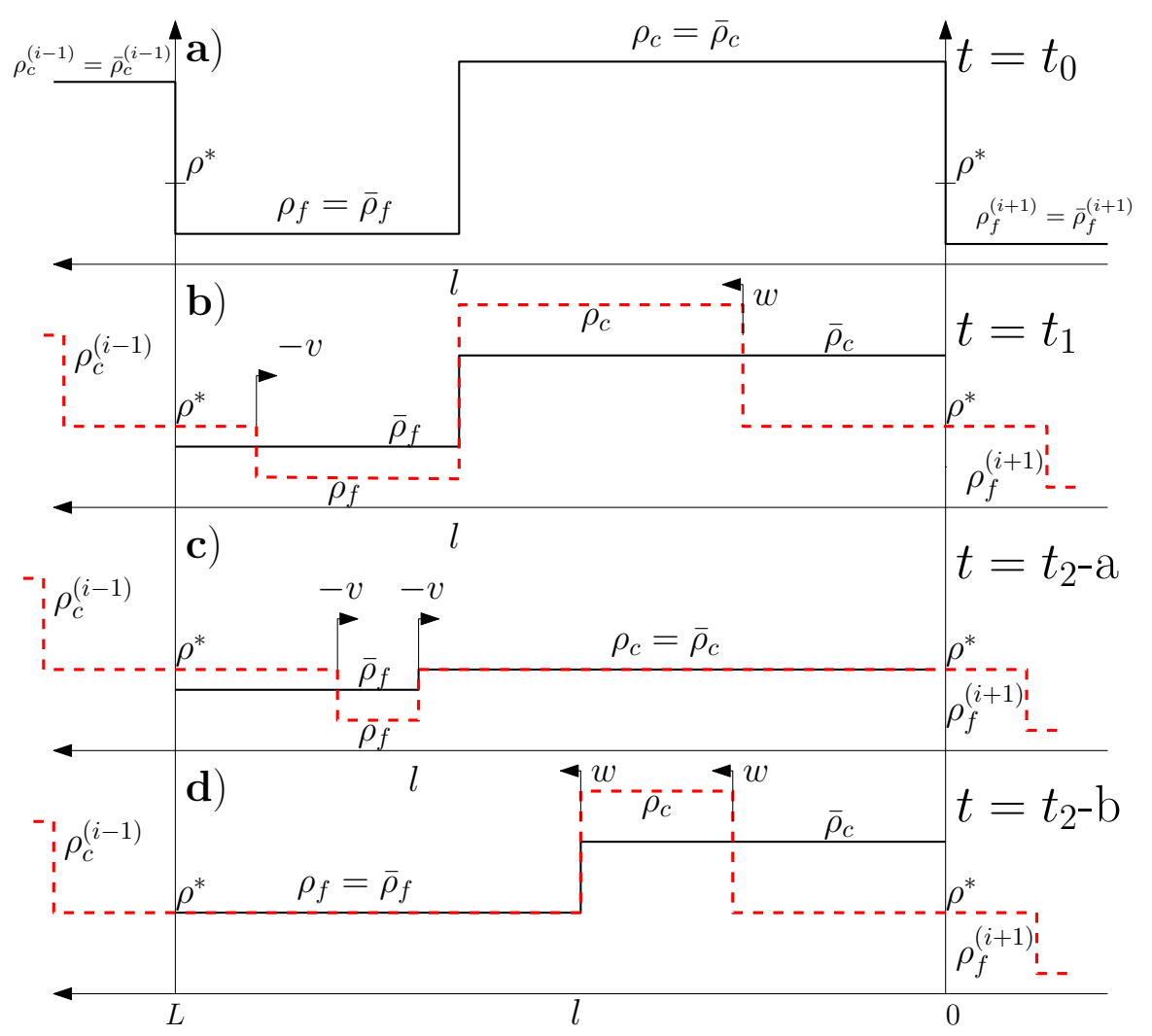

Section $i-1$

Section $i$ Section $i+1$

Fig. 14. Illustration of the density evolution under the effect of the upstream and downstream rarefaction waves. Real solution (solid line), averaged solution (dashed-red line). At $t_{0}$ both solutions coincide before rarefaction waves are produced. At $t=t_{1}$, the rarefaction waves are triggered. At $t=t_{2}$-a, the section becomes fully congested, or alternatively at $t=t_{2}$-b the section becomes fully free depending on which boundary the position $l$ reaches first.

where we have replaced the evolution of $l$, by its definition, $i=f\left(\rho^{-}, \rho^{+}\right)=\frac{\Phi\left(\rho^{+}\right)-\Phi\left(\rho^{-}\right)}{\rho^{-}-\rho^{+}}$. It remains to be defined the interface flows $\left(\varphi_{i n}, \varphi_{\text {out }}, \varphi_{I}\right)$ for all the possible cases produced by this scenario, and shown in Fig. 14.

Determination of $\varphi_{\text {in }}, \varphi_{\text {out }}$ is straightforward as it resorts to the case of a fixed interfaces, when interface flows can be computed using the demand/supply formalism as given by the expressions (87)-(77). Note that this general expression perfectly covers also the rarefaction wave case for which maximum output flow is reached. For instance, looking at the upstream densities distributions in Fig. 14-b, the inflow at $l=L$ for the real solution is computed simply by $\varphi_{i n}=\Phi\left(\rho^{*}\right)=\varphi_{M}$, and for the "approximated" solution, with $\alpha=1, \rho^{*} \leq \bar{\rho}_{c}^{(i-1)} \leq \rho_{M}$, and 
$0 \leq \bar{\rho}_{f}^{(i)} \leq \rho^{*}$, by

$$
\varphi_{i n}=\min \left\{D\left(\bar{\rho}_{c}^{(i-1)}\right), S\left(\bar{\rho}_{f}^{i}\right)\right\}=\min \left\{\varphi_{M}, \varphi_{M}\right\}=\varphi_{M}
$$

Determination of $\varphi_{I}$ is more involved and requires the analysis of several separated cases. There is a simple case where there are not rarefaction waves, where congestion front velocity is typically given by the well-known shown wave speed. This case is accounted by setting $\left(\rho^{-}, \rho^{+}\right)=\left(\bar{\rho}_{f}, \bar{\rho}_{c}\right)$. For other cases when a rarefaction wave is created, it is instructive to look at Fig. 14, in particular for the case shown in Fig. 14-b.

Consider first the case of a single rarefaction wave produced downstream $\left(\alpha^{(i-1)}=0\right.$, and $\left.\alpha^{(i)}=1\right)$. In this case, $\rho_{f}=\bar{\rho}_{f}$, and $\rho_{c} \neq \bar{\rho}_{c}$. In this notation, we understand $\rho_{c}(x)$ in general as a distribution and not as a scalar, except for its initial distribution which is constant before the rarefaction wave start, i.e. $\rho_{c}(x, 0)=\rho_{c}\left(t_{k}\right)=\bar{\rho}_{c}$. It is worth noting that during all the phases shown in Fig. 14-b until the rarefaction wave is fully absorbed (from time $t_{1}$ till $t_{2}-a$, or $t_{2}-b$ ), the evolution of $i$ is dictated by the initial value $\rho_{c}\left(t_{k}\right)$ as the right density $\rho^{+}$remains constant during all this phase. The evolution of $l$ is then given by

$$
i=f\left(\rho_{f}, \rho_{c}\right)=f\left(\bar{\rho}_{f}, \rho_{c}\left(t_{k}^{i}\right)\right)=f\left(\bar{\rho}_{f}, \bar{\rho}_{c}\left(t_{k}^{i}\right)\right)
$$

which corresponds to the first value at the right-hand side of (78), and allows one to have an autonomous equation independent from the "real solution" as it is expected.

The second case where the rarefaction wave is produced only at the upstream boundary $\left(\alpha^{(i-1)}=1\right.$, and $\left.\alpha^{(i)}=0\right)$, is symmetric with respect to the previous one, and the evolution of $l$ can be determined along the same lines, i.e.

$$
i=f\left(\rho_{f}, \rho_{c}\right)=f\left(\rho_{f}\left(t_{k}^{(i-1)}\right), \bar{\rho}_{c}\right)=f\left(\bar{\rho}_{f}\left(t_{k}^{(i-1)}\right), \bar{\rho}_{c}\right)
$$

In third case, we account for rarefaction waves produced at the same time, at both up and downstream boundaries. It basically capture the combination of the previous two solutions.

$$
i=f\left(\rho_{f}, \rho_{c}\right)=f\left(\rho_{f}\left(t_{k}^{(i-1)}\right), \rho_{c}\left(t_{k}^{i}\right)\right)=f\left(\bar{\rho}_{f}\left(t_{k}^{(i-1)}\right), \bar{\rho}_{c}\left(t_{k}^{i}\right)\right)
$$

Finally, in the last case the rarefaction wave will be absorbed and will produce a shock with speed given by the standard Rankine-Hugoniot jump condition. In the case shown in Fig. 14-c, the rarefaction wave from downstream is absorbed first, with the congested density approaching the critical density value, $\rho_{c} \rightarrow \rho^{*}$, and the shock speed being given by $i=-v$. In the other case shown in Fig. 14-d, the rarefaction wave from the upstream is absorbed first, and the free density 
reaches its critical value, $\rho_{f} \rightarrow \rho^{*}$. The shock speed is given by $i=w$. Those computations can be easily verified by noticing that $i$ is nothing but the slope of the triangular characteristic function evaluated at the density of interest. Those cases, as well as the case where there is no rarefaction waves can be coded using the expression given in the last expression of right-hand side of (78).

1) Introduction of the $\epsilon$-boundaries: Handling singularities at the boundaries can be done along the same lines as described in Section IV-D. We then first define the conditions for stopping the time-evolution of the variable $l$. These conditions, $\mathcal{C}_{0}, \mathcal{C}_{L}$ are already given by equation (36) for the downstream boundary, and by (37) for the upstream boundary. The second step consists in defining the interface flows at the respective boundaries: $l=\epsilon$, and $l=L-\epsilon$. As before, those flows are given by the standard demand and supply formulation, $\varphi_{I}=\min \left\{D\left(\rho_{f}\right), S\left(\rho_{c}\right)\right\}$ when one of the two conditions $\mathcal{C}_{0}$, or $\mathcal{C}_{L}$ hold. The last step is the integration of such conditions into the model formulated in Section VII-B, which yields

$$
\begin{aligned}
& \dot{\bar{\rho}}_{f}=\frac{1}{L-l}\left[\varphi_{i n}+f\left(\rho^{-}, \rho^{+}\right)\left(\bar{\rho}_{f}-\rho^{-}\right)-\Phi_{f}\right] \\
& \dot{\bar{\rho}}_{c}=\frac{1}{l}\left[-\varphi_{\text {out }}+f\left(\rho^{-}, \rho^{+}\right)\left(\rho^{+}-\bar{\rho}_{c}\right)+\Phi_{c}\right] \\
& i=f\left(\rho^{-}, \rho^{+}\right)= \begin{cases}\frac{\Phi\left(\rho^{+}\right)-\Phi\left(\rho^{-}\right)}{\rho^{-}-\rho^{+}+\sigma\left(\rho^{-}, \rho^{+}\right)} & \text {else } \\
0 & \text { if } \mathcal{C}_{0} \cup \mathcal{C}_{L}\end{cases} \\
& \Phi_{f}= \begin{cases}\Phi\left(\rho^{-}\right) & \text {else } \\
\min \left\{D\left(\rho_{f}\right), S\left(\rho_{c}\right)\right\} & \text { if } \quad \mathcal{C}_{0} \cup \mathcal{C}_{L}\end{cases} \\
& \Phi_{c}= \begin{cases}\Phi\left(\rho^{+}\right) & \text {else } \\
\min \left\{D\left(\rho_{f}\right), S\left(\rho_{c}\right)\right\} & \text { if } \quad \mathcal{C}_{0} \cup \mathcal{C}_{L}\end{cases}
\end{aligned}
$$

with $\varphi_{\text {in }} \varphi_{\text {out }}, \sigma(\cdot, \cdot)$, and $\rho^{-}, \rho^{+}$as defined before.

\section{Simulation of the urban road with traffic lights}

The simulated scenario concerns a road of three sections of length $L$ with the same fundamental diagram and parameters indicated in Table VII-C. At the end of each section there is a traffic light represented by the variables $\alpha^{i} \in 0,1$. The traffic light sequences are assumed not to be synchronized in order to generate a more complex and illustrative scenario, with rarefaction waves produced at different (non correlated) times. The inflow at the first section (upstream boundary demand) is set to be constant and equal to $\varphi_{\text {in }}^{1}=\Phi\left(\rho_{f}^{0}\right)=2400 \mathrm{veh} / \mathrm{h}$ while the outflow at 
the last section (downstream boundary supply) is set equal to $\varphi_{\text {out }}^{3}=\alpha_{3} \min \left\{D\left(\rho_{c}^{3}\right), S\left(\rho_{f}^{4}\right)\right\}=$ $\alpha_{3} D\left(\rho_{c}^{3}\right)$, i.e. all the demand at the last section is satisfied. A full model with $\epsilon$-boundaries is associated with each section following the mathematical modelling steps previously described. The system is simulated during one hour. The goal of this evaluation is to compare how the model behaves in front to the "real solution" which is simulated using a high resolution CTM (hr-CTM) with very small cell lengths $(5 \mathrm{~m})$. In particular, we will evaluate the evolution of the front congestions (variable $l^{i}$ ) in these two models.

TABLE II

SIMULATION PARAMETERS

\begin{tabular}{|c|c|c|c|}
\hline Parameter & Description & Value & Unit \\
\hline \hline$L$ & section length & 1 & $\mathrm{~km}$ \\
\hline$v$ & free-flow speed & 80 & $\mathrm{~km} / \mathrm{h}$ \\
\hline$w$ & congestion propagation speed & 20 & $\mathrm{~km} / \mathrm{h}$ \\
\hline$\rho_{M}$ & jam density & 250 & $\mathrm{veh} / \mathrm{km}$ \\
\hline$\rho^{*}$ & critical density & 50 & $\mathrm{veh} / \mathrm{km}$ \\
\hline$\varphi_{M}$ & maximum flow & 4000 & $\mathrm{veh} / \mathrm{h}$ \\
\hline$\rho_{f}^{0}$ & initial free-flow cell density & 30 & $\mathrm{veh} / \mathrm{km}$ \\
\hline$\rho_{c}^{0}$ & initial congested cell density & 150 & $\mathrm{veh} / \mathrm{km}$ \\
\hline$l_{0}$ & initial congestion length & {$[0.4 L, 0.4 L, 0.2 L]$} & $\mathrm{km}$ \\
\hline
\end{tabular}

The results are reported in Fig. 15. Note that each subplot shows the evolution of the variable of a different section. It can be observed that the time evolution of the variable $l$ of the proposed VLM suitably tacks the corresponding congestion front of the hr-CTM. Note that the position of this front is not given directly by CTM model but need to be computed by an additional front tracking algorithm added to the CTM in simulation. In the same figures, it can also be observed the time-periods when the light is green (red lines along the time-axis), and the congestion formation just after the out-flows are stopped.

In the next sub-section, we present an illustrative example of the use of the VLM model for control design.

\section{Optimal steady-state speed limits for eco-driving in urban networks}

The problem of finding steady-state speed limits optimizing a trade-off between global traffic energy consumption(Eco-Driving), traveling time and infrastructure utilization, can be can be 

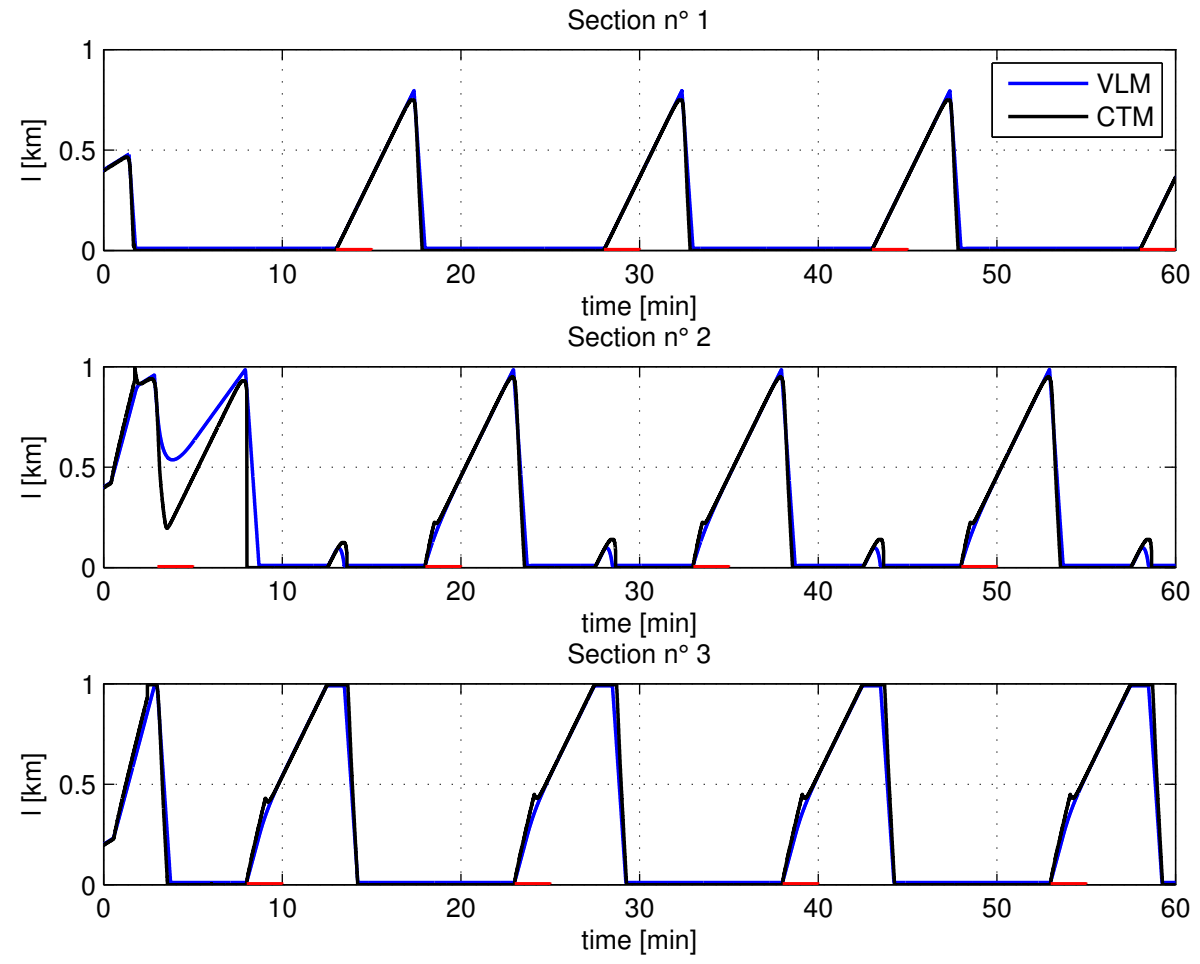

Fig. 15. time evolution of state $l$ of the VLM and congestion front obtained through a hr-CTM.

addressed at macroscopic level using the proposed an averaged version of the two-cells variable length model adapted to the urban setup.

Average model. Let consider the simplest "averaged" version of the model (75)-(78) representing the $i^{\text {th }}$ Section $^{3}$

$$
\begin{aligned}
\dot{\bar{\rho}}_{f} & =\left[\bar{\varphi}_{\text {in }}-\rho_{f} v_{f}\right] \frac{1}{L-l} \\
\dot{\bar{\rho}}_{c} & =\left[w\left(\rho_{m}-\rho_{c}\right)-\bar{\varphi}_{\text {out }}\right] \frac{1}{l} \\
\dot{\bar{l}} & =\frac{\rho_{f} v_{f}-w\left(\rho_{m}-\rho_{c}\right)}{\rho_{c}-\rho_{f}}
\end{aligned}
$$

${ }^{3}$ For simplicity of the notation, the current section index $i$ is drop. When needed the index of the upstream and the downstream section are noted by the subscripts $(i-1)$, and $(i+1)$, respectively. 
whith the corresponding "averaged" boundary flows are defined as:

$$
\begin{aligned}
\bar{\varphi}_{\text {in }} & =\bar{\alpha}^{(i-1)} \cdot \min \left\{D\left(\bar{\rho}_{c}^{(i-1)}\right), S\left(\bar{\rho}_{f}^{i}\right)\right\} \\
\bar{\varphi}_{\text {out }} & =\bar{\alpha}^{i} \cdot \min \left\{D\left(\bar{\rho}_{c}^{i}\right), S\left(\bar{\rho}_{f}^{(i+1)}\right)\right\}
\end{aligned}
$$

the signification of the "average" model here refers to the solutions of the equations above whith the time-varying bi-valued variable $\alpha(t) \in\{0,1\}, \forall t \in\left[t, t+T_{\text {cycle }}\right]$ replaced by its average over a traffic light time-cycle $T_{\text {cycle }}$, i.e.

$$
\bar{\alpha}=\frac{1}{T_{\text {cycle }}} \int_{t}^{t+T_{\text {cycle }}} \alpha(\tau) \mathrm{d} \tau=\frac{T_{\mathrm{gr}}}{T_{\text {cycle }}}
$$

This simplification is also consistent with the store-and-forward modeling approach [1]. The continuous-time behavior of the system, induced by the traffic lights, is then replaced by a continuous flow passing through a bottleneck (with a "capacity drop" (in average) proportional to $\bar{\alpha}$ ), as long as demand and supply functions of upstream and downstream cells, respectively, are capacious enough. As a natural consequences of this approximation, no oscillations of the congestion length, due to the green/red alternation, are represented by the model, and the notions of cycle time and offset for the traffic lights lose significance, see more details at [15] and [10].

Steady-state operation points. Holding the hypothesis of constant and equal boundary flows smaller than maximum flow, such a system converges to the following equilibrium states:

$$
\begin{aligned}
\rho_{f}^{*} & =\frac{\bar{\varphi}_{\text {in }}}{v_{f}} \\
\rho_{c}^{*} & =\rho_{m}-\frac{\bar{\varphi}_{\text {out }}}{w} \\
l^{*} & =\frac{N_{0}-\rho_{f}^{*} L}{\rho_{c}^{*}-\rho_{f}^{*}}=\frac{w v_{f} N_{0}-\bar{\varphi}_{\text {in }} L w}{\left.w v_{f} \rho_{m}-\bar{\varphi}_{\text {out }} v_{f}-\bar{\varphi}_{\text {in }} w\right)}
\end{aligned}
$$

where $N_{0}$ is the initial number of total vehicles in the whole cell. and it will remain unchanged given the assumption of equal boundary flows. Assuming that the speed limit $v_{f}$ is the same both upstream and downstream, then the boundary flows are only determined by the maximum value of the demand/supply function: $\bar{\varphi}_{i n}=\bar{\varphi}_{\text {out }}=\bar{\alpha} \frac{w \rho_{m}}{v_{f}+w} v_{f}$ which allows for a more convenient written of the equilibrium, as an explicit function of the speed limit to be optimized, $v_{f}$, i.e.,

$$
\begin{aligned}
\rho_{f}^{*} & =\bar{\varphi}_{\text {out }}=\bar{\alpha} \frac{w \rho_{m}}{v_{f}+w} \\
\rho_{c}^{*} & =\rho_{m}-\bar{\alpha} \frac{v_{f} \rho_{m}}{v_{f}+w} \\
l^{*} & =\frac{N_{0}\left(v_{f}+w\right)-\bar{\alpha} \rho_{m} L w}{\rho_{m}\left(v_{f}+w\right)(1-\bar{\alpha})}
\end{aligned}
$$


Traffic metrics. Informative traffic metrics assessing traffic and vehicles performance in terms of traveling time, infrastructure utilization and energy consumption, can be adapted to the average variable length traffic model.

Instantaneous Travel Time (ITT) could be simply defined, in the VLM coordinates, as:

$$
\operatorname{ITT}(\rho)=\frac{L-l}{v_{f}}+\frac{l}{v_{c}}
$$

with $v_{c}=-w\left(1-\frac{\rho_{m}}{\rho_{c}}\right)=$. Similarity, the Total Travel Distance (TTD) in steady-state is, is a measure of how efficiently the infrastructure is used in terms of occupancy and traveling velocity. It is defined and adapted to VLM in this framework, evaluated over the traffic light cycle time, as:

$$
\operatorname{TTD}(\rho)=T_{\text {cycle }}\left\{v_{f} \rho_{f} L+\left[w\left(\rho_{m}-\rho_{c}\right)-v_{f} \rho_{f}\right] l\right\}
$$

Energy metrics. Another important metric, usually not considered at macroscopic level, is the energy consumption of the vehicles. The energy cost functional, over a time horizon $T_{\text {cycle }}$, for one section for the VLM can be formulated as follows (see [15] for details).

$$
E=E_{f}+E_{c}
$$

where $E_{f}$, and $E_{c}$ are the energy consumptions in steady state in the free and congested cells, respectively. Minimizing the "average" energy per cycle under constant velocity and steady-state model values, then then respective energy consumption per cycle $\bar{P}(v)$ become a static function of the velocity.The energy functions at the different states of the section can be now computed as follows.

$$
E_{f}=T_{\text {cycle }} \bar{P}_{f}\left(v_{f}\right) \cdot \rho_{f}(L-l)
$$

where $\rho_{f}(L-l)$ is the average number of vehicles in the free part during the traffic light cycle. Similarly, the energy consumption in the congested cell for a constant velocity $\left(v_{c}\right)$ is:

$$
E_{c}=T_{\text {cycle }} \bar{P}_{c}\left(v_{c}\right) \cdot \rho_{c} l
$$

$\bar{P}(v)$ is in general quadratic in $v$. It specific form depends on depends on the type of vehicle considered.

Optimal steady-state operation. Considering admissible demands in a road section, the system is stable and many different equilibrium points can be reached via variable speed limits. The problem can be stated as follows: 

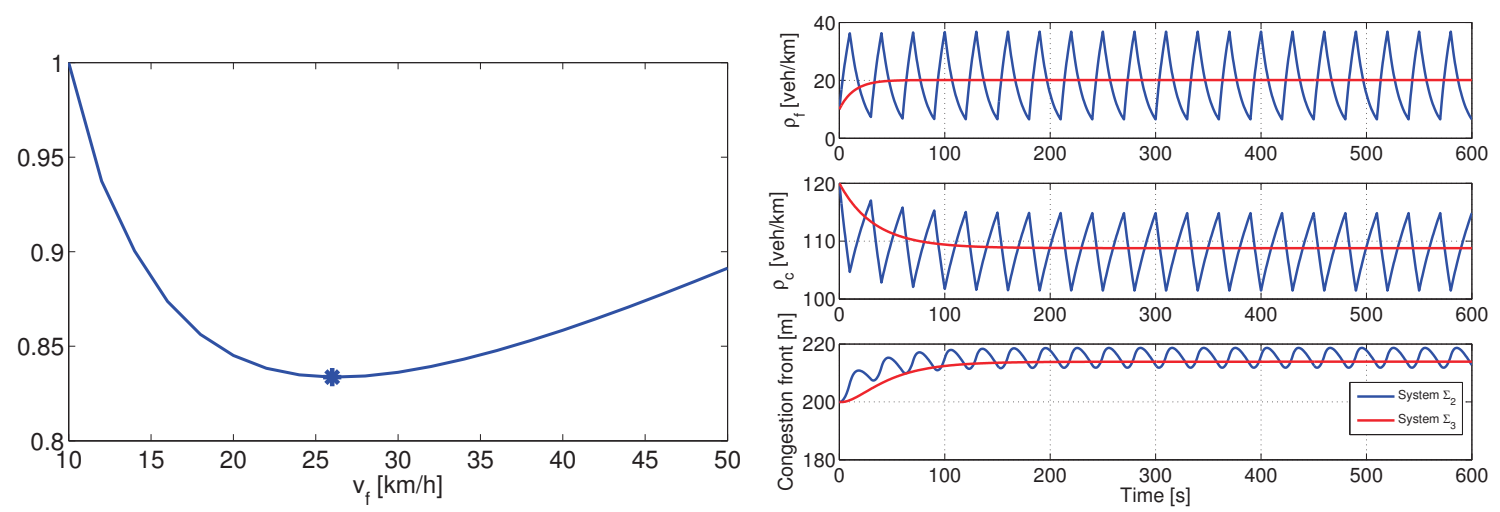

Fig. 16. Left) Total cost function of Problem 2 for system $\Sigma_{3}$ and for an arbitrary choice of weights $\sigma$. A minimum is found for a value $v_{f}^{*}$ of velocity in the free cell. Right) Time evolution of the two systems $\Sigma_{2}$ and $\Sigma_{3}$ for the optimal value of velocity $v_{f}^{*}$.

Problem 1 Given system $\Sigma_{3}$ and a constant $\bar{\alpha}$ for every traffic light, find the optimal speed limit

$$
v_{f}^{*}=\underset{v_{f}}{\operatorname{argmin}}\left\{E+\sigma_{1} I T T-\sigma_{3} T T D\right\}
$$

under: equilibrium steady-sate set (93), and

$$
\begin{aligned}
v_{f} \in \mathcal{U}_{v} & =\left\{v_{f}: v_{\min } \leq v_{f} \leq v_{\max }\right\} \\
l \in \mathcal{U}_{l} & =\left\{l: l_{\text {min }} \leq l \leq l_{\max }\right\}
\end{aligned}
$$

Making the weights choice $\left[\sigma_{1}, \sigma_{3}\right]=[1.2,0.2]$, it is found that $v_{f}^{*}=26 \mathrm{~km} / \mathrm{h}$ (see Fig.(16), left figure) solves the above optimization problem, resulting in a reduction of energy consumption of about $29 \%$, paying in terms of ITT which increases by $27 \%$ and of TTD which decreases by $22 \%$, with respect to the case $v_{f}=v_{\max }$. The total objective function is reduced by $17 \%$ w.r.t. the worst choice of $v_{f}$, and by $6.5 \%$ w.r.t. the standard speed limit of $50 \mathrm{~km} / \mathrm{h}$.

It is natural at this point to apply the optimal velocity $v_{f}^{*}$ for the average system $\Sigma_{3}$, to the original system $\Sigma_{2}$ in which the traffic lights are modeled with the switching variable $\alpha$. Imposing the same hypothesis of equal boundary flows, achievable by using traffic lights with same phase and cycle times, it is possible to compare the time evolution of the state variables and to note how the average system tracks closely the true average of (see Fig.16 right figure). For more details, see [15]. 


\section{CONCLUSIONS}

A new macroscopic vehicular traffic model, named Variable-Length cell transmission Model (VLM), has been presented in this paper. It consists of only three lumped state variables: two lumped densities, associated with the two cells of variable length into which the considered road section is subdivided, and a third state variable which describes the position of the congestion wave front. The paper provides a rigorous formulation of the new traffic model, as well as a detailed analysis of its mathematical properties. As highlighted in the analysis, the VLM naturally encompasses shock waves representation, while it is suitably extended to be able to capture also the phenomenon of wave rarefaction. A comparison with the solution to the classical LWR traffic flow model and the standard Cell Transmission Model has been provided.

Two case studies, a ring road and a urban road with traffic lights, have been considered and thoroughly discussed. First we have illustrated how the VLM can be conveniently exploited to verify the existence of different classes of equilibrium points. Then, we have shown how the proposed model can be used as a tool to facilitate the design of control strategies to optimize the traffic systems operations.

The proposed model can be regarded as a valid alternative to well-established macroscopic models whenever model simplicity is a strict requirement. Apart from being appropriate for optimal speed control design based on the analysis of the equilibria, as seen in the considered case studies, it can be exploited to develop low complexity observers and shockwave front tracking algorithms. The aggregated, low complexity nature of the VLM can give advantage also in traffic forecast and monitoring activities which require the real-time usage of the model.

\section{ACKNOWLEDGEMENTS}

Authors would like to acknowledge Tomas Pippia for helping with the simulations of this paper.

\section{REFERENCES}

[1] K. Aboudolas, M. Papageorgiou, and E. Kosmatopoulos. Store-and-forward based methods for the signal control problem in large-scalecongested urban road networks. Transportation Research Part C: Emerging Technologies, 17 (2). pp. 163-174, 2009.

[2] K. Aboudolas, M. Papageorgiou, A. Kouvelas, and E. Kosmatopoulos, A rolling-horizon quadratic-programming approach to the signal control problem in large-scale congested urban road networks. Transportation Research, Part C 17, 680-694, 2009. 
[3] Balijepalli, N.C., Ngoduy, D., Watling, D.P. The two-regime transmission model for network loading in dynamic traffic assignment problems. Transportmetrica A: Transport Science 10, 2014. 563-584. doi:10.1080/18128602.2012.751680

[4] L.D. Baskar, B. De Schutter, and H. Hellendoorn: Dynamic speed limits and on-ramp metering for IVHS using model predictive control, Proceedings of the 11th International IEEE Conference on Intelligent Transportation Systems (ITSC 2008), Beijing, China, pp. 821-826, Oct. 2008.

[5] Bliemer, M. Dynamic Queuing and Spillback in Analytical Multiclass Dynamic Network Loading Model. Transportation Research Record: Journal of the Transportation Research Board 2029, 14-21. 2007. doi:10.3141/2029-02

[6] Bliemer, M. C., Raadsen, M. P., Brederode, L. J., Bell, M. G., Wismans, L. J., Smith, M. J.: Genetics of traffic assignment models for strategic transport planning. Transport reviews, 37(1), 56-78, 2017.

[7] M. Brackstone, and B. Sultan, M. McDonald: Motorway driver behaviour: studies on car following, Transportation Research Part F, pp. 31-46, Vol.5, 2002.

[8] M. Burger, M. van den Berg, A. Hegyi, B. De Schutter, J. Hellendoorn: : Considerations for model-based traffic control, Transportation Research Part C: Emerging Technologies, Volume 35, 2013, Pages 1-19

[9] C. Canudas-de-Wit: Best-effort Highway Traffic Congestion Control via Variable Speed Limits, 50th IEEE Conference on Decision and Control and European Control Conference (IEEE CDC-ECC 2011), Orlando, Floride, United States. Dec 2011.

[10] C. Canudas-de-Wit: An Average Study of the Signalized Cell Transmission Model, IEEE Transactions on Automatic Control, 2015. Under review.

[11] C. Canudas-de-Wit, F. Morbidi, L. Ojeda, A. Kibangou, I. Bellicot and P. Bellemain: Grenoble Traffic Lab: An Experimental Platform for Advanced Traffic Monitoring and Forecasting, IEEE Control Systems Magazine, Vol.35, pp.23-39, June 2015.

[12] J.R.D. Frejo, E.F. Camacho: Global Versus Local MPC Algorithms in Freeway Traffic Control With Ramp Metering and Variable Speed Limits, IEEE Transactions on Intelligent Transportation Systems, Vol.13, pp.1556-1565, Dec. 2012.

[13] C. M. Dafermos: Polygonal approximations of solutions of the initial value problem for a conservation law, Journal of Mathematical Analysis and Applications, Vol. 38, pp. 33-41, 1972.

[14] C. F. Daganzo: The Cell Transmission Model: A Dynamic Representation of Highway Traffic Consistent with the Hydrodynamic Theory, Transportation Research Board, 28(4), 269-287, 1994.

[15] G. De Nunzio Traffic Eco-Management in Urban Traffic Networks, PhD. Thesis INP, Grenoble France, 2015. https://hal.archives-ouvertes.fr/tel-01225232

[16] A. Ferrara, A. Nai Oleari, S. Sacone, S. Siri: Freeways as Systems of Systems: A Distributed Model Predictive Control Scheme, IEEE Systems Journal, Vol. 9, pp. 312-323, 2015.

[17] A. Ferrara, S. Sacone, S. Siri: Event-triggered model predictive schemes for freeway traffic control, Transportation Research C, Vol. 58, pp. 554?567, 2015.

[18] D. C. Gazis and R. B. Potts: The oversaturated intersection. In: Proc. of the 2nd International Symposium on Traffic Theory. London, UK, pp. 221-237, 1963

[19] S. Godunov: A difference scheme for numerical solution of discontinuous solution of hydrodynamic equations, Matematicheskii Sbornik, Vol. 47, pp. 271-306, 1959.

[20] G. Gomes, R. Horowitz, A. A. Kurzhanskiy, J. Kwon, and P. Varaiya: Behavior of the Cell Transmission Model and Effectiveness of Ramp Metering, Transportation Research C, Vol. 16, pp. 485-513, 2008.

[21] G. Gomes, R. Horowitz: Optimal freeway ramp meetering using the asymetric cell transmission model, Transportation Research, C, Vol. 14, 244-262, 2006.

[22] A. Hegyi, B. De Schutter, and H. Hellendoorn: Model predictive control for optimal coordination of ramp metering and variable speed limits Transportation Research Part C, Vol. 13, pp. 185-209, June 2005. 
[23] R. Herbin, L. Leclercq: A note on the Entropy solutions of the hydrodynamic model of traffic flow revisited, Vol. 45, Issue 1, pp. 138-142, online publication, 2011.

[24] W. Himpe, R. Corthoutb, M.J.C. Tampère: An efficient iterative link transmission model, Transportation Research Part B (2016). Washington, DC.

[25] S. P. Hoogendoorn and P.H.L. Bovy: State-of-the-art of vehicular traffic flow modelling, Proceedings of the Institution of Mechanical Engineers, Part I: Journal of Systems and Control Engineering, Vol. 215, pp. 283-303, 2001.

[26] F. van Wageningen-Kessels, H. van Lint, K. Vuik and S. Hoogendoorn: Genealogy of traffic flow models, EURO Journal on Transportation and Logistics, pp. 1-29, 2014.

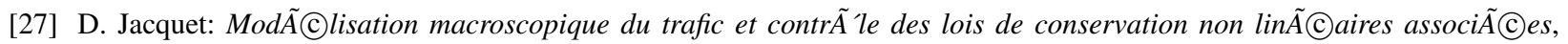
Institut National Polytechnique de Grenoble-INP, Ph.D. Thesis, 2006.

[28] D. Jacquet, C. Canudas De Wit and D. Koenig: Optimal Control of Systems of Conservation Laws and Application to Non-Equilibrium Traffic Control, Proceedings of the 13th IFAC Workshop on Control Applications of Optimization, France 2006.

[29] D. Koenig, D. Jacquet and C. Canudas De Wit: Optimal ramp metering strategy with extended LWR model, analysis and computational methods, Preprints of the 17th IFAC World Congress - IFAC World Congress, Czech Republic, 2005.

[30] J. P. Lebacque. The Godunov scheme and what it means for first order traffic flow models. In Proc. of the International Symposium on Transportation and Traffic Theory, Lyon, France, 1996.

[31] J.P. Lebacque, S. Mammar, H. Haj-Salem, Generic second order traffic flow modelling, In: All- sop, R.E., Bell, M.G.H., Heydecker, B.G. (Eds) Proceedings of the 17th International Symposium on Transportation and Traffic Theory. London: Elsevier, 2007, pp. 755-776.

[32] R.J. LeVeque: Numerical methods for conservation laws, Birkhäuser, 1992.

[33] J. Levy, J. Buonocore and K. von Stackelberg: Evaluation of the public health impacts of traffic congestion: a health risk assessment. Environmental Health, Vol. 9, pp. 65, 2010.

[34] M. J. Lighthill and G. B. Whitham: On kinematic waves II: A theory of traffic flow on long crowded roads, Proceedings of the Royal Society of London. Series A, Mathematical and Physical Sciences, Vol. 229, pp. 317-345, 1955.

[35] W.H. Lin and D. Ahanotu: Validating the Basic Cell Transmission Model on a Single Freeway Link, PATH Technical Note 95-3, Institute of Transportation Studies, University of California at Berkeley, 1994.

[36] P.W. Lin and K.P. Kang, and G.L. Chang: Exploring the effectivness of variable speed limit control on highways work-zone operation. International Journal of Intelligent Transportation Systems, Vol. 8, pp. 155-168, 2004.

[37] M. Patriksson: The traffic assignment problem: models and methods. Courier Dover Publications, 2015.

[38] S. Maerivoet, and B. De Moor: Cellular automata models of road traffic, Physics Reports, Vol. 419, pp. 1-64, 2005.

[39] G.F. Newell: A simplified theory of kinematic waves in highway traffic, Transportation Research 27B, 281-313, 1993.

[40] O. A. Oleinik: On the uniqueness of the generalized solution of the Cauchy problem for a non-linear system of equations occurring in mechanics, Uspekhi Mat. Nauk. Vol. 12, pp. 169-176, 1957.

[41] P. I. Richards: Shock Waves on the Highway, Operations Research, Vol. 4, pp. 42-51, 1956.

[42] M. Saifuzzaman, and Z. Zheng: Incorporating human-factors in car-following models: A review of recent developments and research needs, Transportation Research Part C, Vol. 48, pp. 379-403, 2014.

[43] A. Smadi, J. Baker and S. Birst: Advantages of Using Innovative Traffic Data Collection Technologies. Applications of Advanced Technology in Transportation, ASCE Library, Chapter 102, pp. 641-646, 2006.

[44] M. Treiber and A. Kesting: Traffic flow dynamics - Data, models and simulation, Springer, 2013.

[45] H. van Wageningen-Kessels, H. van Lint, S. Hoogendoorn, and K. Vuik. Genealogy of traffic flows models. EURO J. Transp Logistics 4:445-473, 2015 
[46] I. Yperman, C.M.J. Tampère: Multi-commodity dynamic network loading with kinematic waves and intersection delays. In Proceedings of the DTA, Leeds, UK, 2006.

[47] I. Yperman, S. Logghe, C.M.J. Tampère and L.H. Immers: The Multi-Commodity Link Transmission Model for Dynamic Network Loading. In Proceedings 85th Annual Meeting of the TRB, 2006.

[48] I. Yperman, The link Transmission Model for Dynamic Network Laoding, Ph.D, Katholieke Universiteit Leuven ATHOLIEKE UNIVERSITEIT LEUVEN, June 2007.

[49] P. Varaiya: Max pressure control of a network of signalized intersections. Transportation Research Part C Vol.36, (2013), 177-195. 